\title{
Categorification of the Jones-Wenzl projectors
}

\author{
Benjamin Cooper and Vyacheslav Krushkal ${ }^{1}$
}

\begin{abstract}
The Jones-Wenzl projectors $p_{n}$ play a central role in quantum topology, underlying the construction of $\mathrm{SU}(2)$ topological quantum field theories and quantum spin networks. We construct chain complexes $P_{n}$, whose graded Euler characteristic is the "classical" projector $p_{n}$ in the Temperley-Lieb algebra. We show that the $P_{n}$ are idempotents and uniquely defined up to homotopy. Our results fit within the general framework of Khovanov's categorification of the Jones polynomial. Consequences of our construction include families of knot invariants corresponding to higher representations of $\mathrm{U}_{q} \mathfrak{s l}(2)$ and a categorification of quantum spin networks. We introduce $6 j$-symbols in this context.
\end{abstract}

Mathematics Subject Classification (2010). Primary 57M27; Secondary 18G60, 81R50, $57 \mathrm{M} 25$.

Keywords. Categorification, Jones-Wenzl projectors, spin networks, categorified representation theory, colored Jones polynomial, $6 j$-symbols.

\section{Contents}

1 Introduction . . . . . . . . . . . . . . . . . . . . . . . . . 139

2 The Temperley-Lieb algebra and the Jones-Wenzl projectors . . . . . . 142

3 Universal projectors and statement of the main theorem . . . . . . . 150

4 Explicit formulae and computations . . . . . . . . . . . . . 152

5 Reidemeister moves and graphical calculus . . . . . . . . . . . . . 156

6 Spin networks . . . . . . . . . . . . . . . . . . . 158

7 Proof of the main theorem . . . . . . . . . . . . . . . . . . 164

References . . . . . . . . . . . . . . . . . . . . . 178

\section{Introduction}

In [15] Mikhail Khovanov introduced a categorification of the Jones polynomial, giving rise to a new conceptual framework for quantum invariants of links in the

\footnotetext{
${ }^{1}$ The second author was partially supported by the NSF and by the IHES.
} 
3 -sphere. The results in [15] fit in the context of categorification of the TemperleyLieb algebra; see [16] and [4] (also [6] and [24]). Roughly speaking, categorification associates to an algebra $A$ a category $\ell$ whose Grothendieck group $\mathrm{K}_{0}(\mathcal{C})$ is isomorphic to $A$. Moreover, multiplication by generators of $A$ gives rise to functors acting on $\mathcal{C}$ and satisfying natural properties [19]. An extension from planar Temperley-Lieb diagrams to tangles is achieved by passing from additive to triangulated categories. The resulting link homology theory satisfies functoriality under surface cobordisms in 4-space, an important feature that was not apparent at the level of its graded Euler characteristic, the Jones polynomial.

An important open problem in the subject is to extend known categorifications from links in the 3-sphere to quantum invariants of 3-manifolds. The constructions of the SU(2) quantum invariants by Reshetikhin-Turaev [25] and Turaev-Viro [26] rely on the Jones-Wenzl projectors $p_{n}$, certain special elements of the Temperley-Lieb algebra; see [13] and [29]. In the Reshetikhin-Turaev theory, one uses the JonesWenzl projectors to label the components of the link in a surgery presentation of the 3-manifold. In the Turaev-Viro approach, to a triangulation of the 3-manifold is assigned a state sum involving the $6 j$-symbols, an important ingredient in the theory of quantum spin networks. (An additional key feature of 3-manifold invariants, closely related to the properties of the Jones-Wenzl projectors, is that the "quantum" parameter $q$ has to be specialized to a root of unity in order to get a semisimple theory).

The main goal of this paper is to introduce a categorification of the Jones-Wenzl projectors. The Temperley-Lieb algebra $\mathrm{TL}_{n}$ is an algebra over $\mathbb{Z}\left[q, q^{-1}\right]$, additively generated by planar diagrams connecting $n$ points at the top and at the bottom of a rectangle and the multiplication is defined on generators by vertical stacking of such diagrams (see Section 2 below for more details). The Jones-Wenzl projector $p_{n}$ is an idempotent element of $\mathrm{TL}_{n}$, uniquely characterized by the following two properties: (1) the coefficient of the unit element, corresponding to $n$ vertical strands, in the expression for $p_{n}$ is 1 and (2) $p_{n}$ is "killed by turnbacks", that is $p_{n} D=D p_{n}=0$ where $D$ is any planar diagram generator of $\mathrm{TL}_{n}$ other than the unit element.

Note that, unlike the Jones polynomial and various other link invariants that have been previously categorified, the coefficients in the expansion of $p_{n}$ in terms of the generators of $\mathrm{TL}_{n}$ are rational, rather than polynomial, functions of $q, q^{-1}$. This suggests that categorification of the projectors cannot be achieved by chain complexes of finite length.

We use Bar-Natan's formulation of Khovanov's theory: the objects in this category are the Temperley-Lieb diagrams and morphisms are surface cobordisms in 3-space between such diagrams; see [4] and Section 2 below. In this framework, for each $n$ we construct a chain complex $P_{n}$ whose graded Euler characteristic is the formal power series corresponding to $p_{n}$. For example, the power series for $n=2$ is

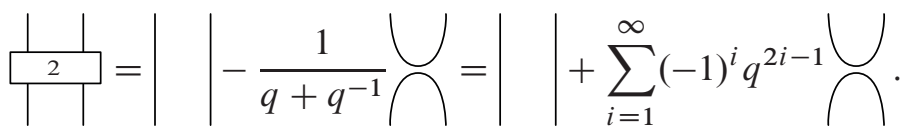


We show that the chain complexes $P_{n}$ are uniquely characterized up to homotopy by properties analogous to those of the Jones-Wenzl projectors $p_{n} \in \mathrm{TL}_{n}$ : (1) the identity diagram appears in the chain complex $P_{n}$ only once, in degree zero and (2) $P_{n}$ is contractible "under turnbacks"; see the definition of a universal projector and Theorem 3.2 in Section 3. It follows from these properties that $P_{n}$ is a "homotopy idempotent": $P_{n} \otimes P_{n} \simeq P_{n}$. We write down the chain complexes explicitly for $n=2,3$, see Section 4. The main technical part of the paper is the inductive construction of the chain complex $P_{n}$ for larger $n$ in Section 7, modeled on the Frenkel-Khovanov recursion [7] for the Jones-Wenzl projectors. The universality properties satisfied by $P_{n}$ and the invariance under Reidemeister moves, discussed further below, suggest the naturality of the construction proposed in this paper. We summarize the discussion so far with the table below.

$\begin{array}{ll}\text { Algebra } & \frac{\text { Category }}{\text { Temperley-Lieb algebra: } \mathrm{TL}_{n}} \\ p_{n} \in \mathrm{TL}_{n} & \text { Bar-Natan-Khovanov Category: } \operatorname{Kom}(n) \\ p_{n} \cdot p_{n}=p_{n} & P_{n} \in \operatorname{Kom}(n), K_{0}\left(P_{n}\right)=p_{n} \\ p_{n} \text { is unique } & P_{n} \otimes P_{n} \simeq P_{n} \\ & P_{n} \text { is unique up to homotopy }\end{array}$

An immediate consequence of our construction is a categorification of quantum spin networks. That is, to a spin network $G$ we associate a chain complex whose graded Euler characteristic is a Laurent series in $q$ corresponding to the quantum evaluation of $G$. Some interesting phenomena are observed here. In the simplest example, the rational homology of the trace of the second projector, $\operatorname{Tr}\left(P_{2}\right)$, has the expected graded Euler characteristic [3] $=q^{-2}+1+q^{2}$, but the homology itself has infinite rank (with extra generators canceling in pairs in the Euler characteristic). Further, there is 2-torsion when the homology is taken with integer coefficients; see Section 4.1.1. In Section 6.2 we formulate a categorified analogue of the $6 j$-symbols. It takes the form of an iterated cone construction, giving rise to a "homotopy change of basis" in the category of chain complexes.

Our construction also gives rise to an invariant of tangles, leading to a categorification of the colored Jones polynomial, see Section 5. Note that the included computations imply that our work is different from the previously defined categorification of the colored Jones polynomial [17] (see also [5]). See Section 4.1 for further discussion.

We would like to mention that, while preparing this manuscript for publication, during the MSRI workshop "Homology Theories of Knots and Links" in March 2010 we learned that an alternative, representation-theoretic, approach to categorifying the Jones-Wenzl projectors has been pursued by Igor Frenkel, Catharina Stroppel and Joshua Sussan [9]. In light of the universality properties of our construction (see Section 3), it is plausible that the two approaches are equivalent, although the methods are quite different. One advantage of working in Khovanov's and BarNatan's framework for categorification of the Temperley-Lieb algebra is that our 
construction of the categorified projectors is explicit and it is readily available for topological applications. The interested reader may want to compare our construction in Section 6.2 to the discussion of the $6 j$-symbols in [9], Section 17.

We would like to add that more recently Lev Rozansky [23] has proposed an elegant idea on categorification of the Jones-Wenzl projectors, based on the properties of the infinite torus braid. Our construction is based on the Frenkel-Khovanov recursive formula, however it seems reasonable to believe that the two approaches may be related (and more generally the universality properties satisfied by the projectors imply that the different constructions are homotopy equivalent).

Acknowledgements. The authors would like to thank the referee for a number of suggestions which have led to a better exposition.

\section{The Temperley-Lieb algebra and the Jones-Wenzl projectors}

This section summarizes the relevant background on the definition and categorification of the Temperley-Lieb algebra. Section 2.4 states a version of the Gaussian elimination lemma which will be used throughout the paper.

2.1. The Temperley-Lieb algebra. We recall that the Temperley-Lieb algebra is the unital $\mathbb{Z}\left[q, q^{-1}\right]$-algebra of $\mathrm{U}_{q} \mathfrak{s l}(2)$-equivariant maps between $n$-fold tensor powers of the fundamental representation $V$,

$$
\mathrm{TL}_{n}=\operatorname{Hom}_{\mathrm{U}_{q} \mathfrak{s l}(2)}\left(V^{\otimes n}, V^{\otimes n}\right) .
$$

There is an explicit presentation given by the standard generators 1 and $e_{i}, 0<i<n$, satisfying the relations:

(1) $e_{i} e_{j}=e_{j} e_{i}$ if $|i-j| \geq 2$,

(2) $e_{i} e_{i \pm 1} e_{i}=e_{i}$,

(3) $e_{i}^{2}=[2] e_{i}$,

where the quantum integer $[n]$ is defined to be

$$
[n]=\frac{q^{n}-q^{-n}}{q-q^{-1}}=q^{-(n-1)}+q^{-(n-3)}+\cdots+q^{n-3}+q^{n-1} .
$$

Each generator $e_{i}$ can be pictured as a diagram consisting of $n$ chords between two collections of $n$ points on two horizontal lines in the plane. All strands are vertical except for two, connecting the $i$ th and the $(i+1)$-st points in each collection. For instance, when $n=3$ we have the following diagrams,

$$
1=||, \quad e_{1}=\bigcup \mid, \quad \text { and } e_{2}=\mid \bigcap .
$$


The multiplication is given by vertical composition of diagrams, and planar isotopy induces relations (1) and (2) between the generators above. The third relation says that any circles which are created may be removed at the cost of multiplication by $[2]=q+q^{-1}$.

This algebra is well-known in low-dimensional topology in particular due to its natural extension from planar diagrams to tangles, captured by the Kauffman bracket relations

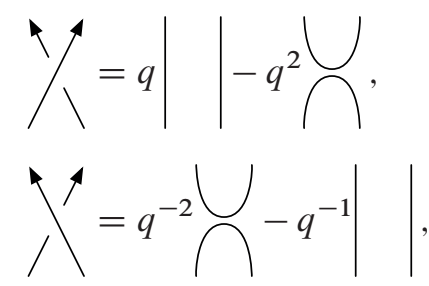

which yield the Jones polynomial up to normalization; see [14] and [13].

2.2. Jones-Wenzl projectors. The Jones-Wenzl Projectors $p_{n} \in \mathrm{TL}_{n}$ are idempotent elements of the Temperley-Lieb algebra which have proven to be fundamental to its study and applications. The projectors appear in the study of spin networks or the graphical calculus of higher $\mathrm{U}_{q} \mathfrak{s l}(2)$ representations, the colored Jones polynomial and many constructions of Chern-Simons theory; see [14], [26], [25], [2], [3], and [27].

The projectors were originally [29] defined by the recurrence relation

$$
\begin{aligned}
& p_{1}=1, \\
& p_{n}=p_{n-1}-\frac{[n-1]}{[n]} p_{n-1} e_{n-1} p_{n-1} .
\end{aligned}
$$

If we depict $p_{n}$ graphically by a box with $n$ incoming and outgoing chords:

$$
p_{n}=\underset{\mid \uparrow}{\downarrow},
$$

then the formula may be illustrated as follows:

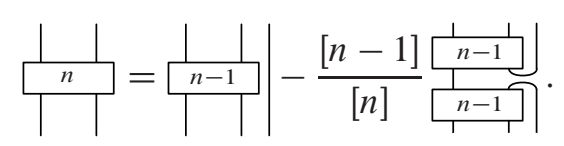

It can be shown that the Jones-Wenzl projectors are uniquely characterized by the following properties:

(1) $p_{n} \in \mathrm{TL}_{n}$ considered as a $\mathbb{Z}\left[q^{-1}\right] \llbracket q \rrbracket$-algebra;

(2) $p_{n}-1$ belongs to the subalgebra generated by $\left\{e_{1}, e_{2}, \ldots, e_{n-1}\right\}$; 
(3) $e_{i} p_{n}=p_{n} e_{i}=0$ for all $i=1, \ldots, n-1$.

See for instance [14] and [21].

The coefficients of Temperley-Lieb diagrams in the expression for $p_{n}$ uniquely determine power series with positive powers of $q$. The equations above then define $p_{n}$ as a power series in Temperley-Lieb elements, for example the power series for $p_{2}$ is given in (1) in the introduction. (Alternatively, one could expand rational functions as series in $q^{-1}$ producing a dual projector, see discussion following Definition 3.1.)

2.3. Categorification of the Temperley-Lieb algebra. Work by a number of authors on the existence of integral bases in Lie theory led to a categorification of the Temperley-Lieb algebra by Mikhail Khovanov in which integer coefficients were interpreted as the dimensions of graded vector spaces and polynomials as graded Euler characteristics; see [7], [1], [15], [16], [24], and [8]. This construction extends to tangles and there is a corresponding functoriality with respect to cobordisms between these tangles; see [12] and [4].

In this section we recall Dror Bar-Natan's graphical formulation [4] of the Khovanov categorification. It will be used throughout the remainder of this paper. Using the Bar-Natan formulation has the advantage of allowing our constructions to apply to a number of variant categorifications which exist in the literature.

Definition 2.1. If $\mathcal{C}$ is an additive category then the split Grothendieck group of $\mathcal{C}$ is

$$
K_{0}(\mathcal{C})=\mathbb{Z}\langle\operatorname{Iso}(\mathcal{C})\rangle /([A \oplus B]=[A]+[B]),
$$

the free abelian group generated by isomorphism classes of objects in $\mathcal{C}$ modulo the relation above. If $\mathcal{C}$ is a monoidal category then the map $\mathcal{C} \otimes \rightarrow \mathcal{C}$ induces $K_{0}(\mathcal{C}) \otimes K_{0}(\mathcal{C}) \rightarrow K_{0}(\mathcal{C})$, endowing $K_{0}(\mathcal{C})$ with an algebra structure; see [28] and [19].

Our goal is to define an additive monoidal category $\operatorname{Cob}(n)$ such that

$$
K_{0}(\operatorname{Cob}(n)) \cong \mathrm{TL}_{n} .
$$

There is an additive category $\operatorname{Pre-Cob}(n)$ whose objects are isotopy classes of formally $q$-graded Temperley-Lieb diagrams with $2 n$ boundary points. The morphisms are given by the free $\mathbb{Z}$-module spanned by isotopy classes of orientable cobordisms bounded in $\mathbb{R}^{3}$ between any two planes containing such diagrams.

The degree of a cobordism $C: q^{i} A \rightarrow q^{j} B$ is given by

$$
\operatorname{deg}(C)=\operatorname{deg}_{t}(C)+\operatorname{deg}_{q}(C),
$$

where the topological degree $\operatorname{deg}_{t}(C)=\chi(C)-n$ is given by the Euler characteristic of $C$ and the $q$-degree $\operatorname{deg}_{q}(C)=j-i$ is given by the relative difference in $q$-gradings. The maps $C$ used throughout the paper will satisfy $\operatorname{deg}(C)=0$. The 
formal $q$-grading will be chosen to cancel the topological grading. When working with chain complexes every object will also contain a homological grading and every map will have an associated homological degree. Homological degree is not part of the definition $\operatorname{deg}(C)$.

It has become a common notational shorthand to represent a handle by a dot and a saddle by a flattened diagram containing a dark line:

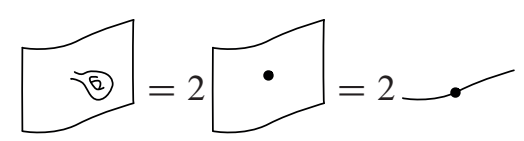

and

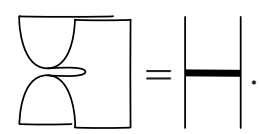

(The topological degrees of the cobordisms above are $-2,-1$ respectively.)

We would like a category $\ell$ such that $\mathrm{K}_{0}\left(\mathcal{\ell} \cong \mathrm{TL}_{n}\right.$ so we require that the object represented by a closed circle be isomorphic to sum of two empty objects in degrees \pm 1 respectively. If such maps are to be degree preserving then the most natural choice for these

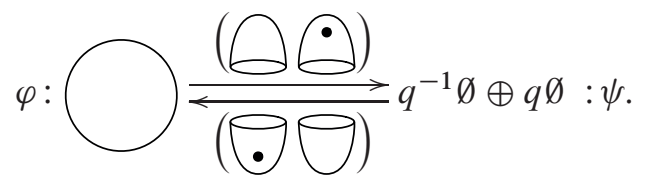

In order to obtain $\varphi \circ \psi=1$ and $\psi \circ \varphi=1$ we form a new category $\operatorname{Cob}(n)=$ $\mathrm{Cob}_{\cdot / l}^{3}(n)$ obtained as a quotient of the category Pre-Cob $(n)$ by the relations given below:

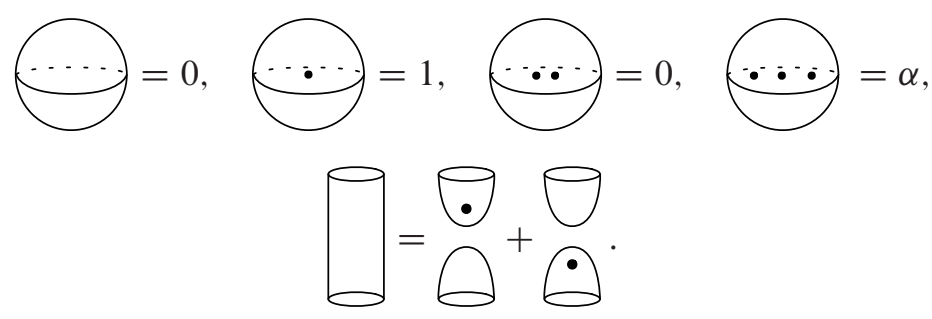

The cylinder or neck cutting relation implies that closed surfaces $\Sigma_{g}$ of genus $g>3$ evaluate to 0 . In what follows we will let $\alpha$ be a free variable and absorb it into our base ring $\left(\Sigma_{3}=8 \alpha\right)$. One can think of $\alpha$ as a deformation parameter; see [4] for further details.

Example 2.2 (the circle). In both Pre-Cob(0) and $\mathrm{Cob}(0)$ there are objects associated to the circle and the empty set. Consider

$$
A=\operatorname{Hom}_{\mathrm{Pre}-\mathrm{Cob}(0)}(\varnothing, \bigcirc) \text { and } B=\operatorname{Hom}_{\mathrm{Cob}(0)}(\varnothing, \bigcirc) \text {. }
$$


Both $A$ and $B$ are abelian groups. An element of $A$ consists of a linear combination of isotopy classes of orientable surfaces with a single fixed boundary circle. An element of $B$ consists of a linear combination of such surfaces subject to the relations above. In particular, the last relation allows us to cut any surface along a closed curve which bounds a disk in 3-space. The reader can check that every element $x \in B$ is of the form

$$
x=m \bullet+n \vartheta
$$

for some $m, n$. $\operatorname{Hom}_{\operatorname{Cob}(0)}(\emptyset, \bigcirc)$ can be endowed with a Frobenius algebra structure using maps induced by cobordisms. When $\alpha=0$ this is the Frobenius algebra which appears in Khovanov's original construction; see [18].

Given two objects $C, D \in \operatorname{Cob}(n)$ we will use $C \otimes D$ to denote the map

$$
\operatorname{Cob}(n) \otimes \operatorname{Cob}(n) \longrightarrow \operatorname{Cob}(n)
$$

obtained by gluing all diagrams and morphisms along the $n$ boundary points and $n$ boundary intervals respectively; pictorially we have

$$
C \otimes D=\frac{\begin{array}{l}
1 \\
\frac{1}{1} \\
\frac{1}{1} \\
\hline 1
\end{array} .}{1} \text {. }
$$

Lemma 2.3. There is an isomorphism of $\mathbb{Z}\left[q, q^{-1}\right]$-algebras

$$
K_{0}(\operatorname{Cob}(n)) \cong \mathrm{TL}_{n}
$$

Proof. Note that the $q$-degree shifting functor determines an endomorphism

$$
K_{0}(q): K_{0}(\operatorname{Cob}(n)) \longrightarrow K_{0}(\operatorname{Cob}(n))
$$

making $K_{0}(\operatorname{Cob}(n))$ into a $\mathbb{Z}\left[q, q^{-1}\right]$-algebra. The proof follows directly from the construction above.

The categorifications $\operatorname{Cob}(n)$ fit together in much the same way as the TemperleyLieb algebras $\mathrm{TL}_{n}$. There is an inclusion $-\sqcup 1^{m-n}: \operatorname{Cob}(n) \rightarrow \operatorname{Cob}(m)$ whenever $n \leq m$ obtained by unioning each diagram with $m-n$ disjoint vertical line segments on the right to each object and $m-n$ disjoint disks to each morphism. If $m=n$ then the empty set is used instead of either intervals or disks.

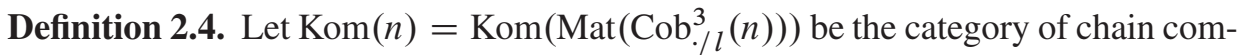
plexes of finite direct sums of objects in $\mathrm{Cob}_{\cdot / l}^{3}(n)$. We allow chain complexes $K_{*}$ of unbounded positive homological degree and require that for each $K_{*}$ there exists an $N \in \mathbb{Z}_{-}$such that $K_{n}=0$ for $n<N$. 
Note that $\otimes$ extends to $\operatorname{Kom}(n)$; see [10]. The functor $-\sqcup 1^{m-n}$ extends to $\operatorname{Kom}(n)$ in the obvious way. The skein relation becomes

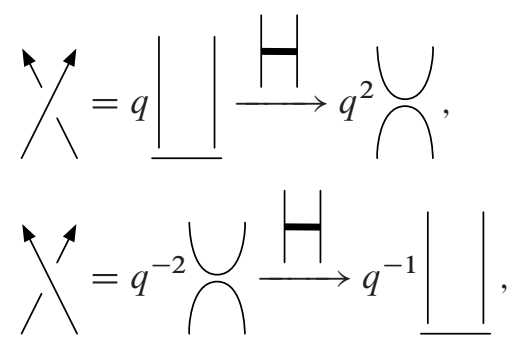

where the underlined diagram represents homological degree 0 .

The skein relation allows us to associate to any tangle diagram $D$ with $2 n$ boundary points an object in $\operatorname{Kom}(n)$.

2.3.1. Grothendieck group of $\operatorname{Kom}(\boldsymbol{n})$. We have included this section in order to explain how the word categorification pertains to the categories of partially unbounded chain complexes appearing in this paper. What appears here represents only a minor variation of the theory; see [28].

Definition 2.5. If $K$ is a finite sum of $q$-graded objects then define $\max _{q}(K)$ to be the maximum $q$-degree of a summand of $K$. Define $\min _{q}(K)$ to be the minimal $q$-degree of a summand of $K$.

Let $\operatorname{Kom}^{0}(n) \subset \operatorname{Kom}(n)$ be the subcategory consisting of chain complexes $K_{*}$ such that the functions $f(i)=\max _{q} K_{i}$ and $g(j)=\min _{q} K_{j}$ are both monotonically increasing.

All of the constructions in this paper reside within this subcategory of $\operatorname{Kom}(n)$. For example, the diagram in Section 7.2, which is important in the proof of the main theorem, has monotonically increasing $q$-degree. It follows from the definition of $\operatorname{Kom}^{0}(n)$ that the $q$-graded Euler characteristic of any object $C \in \operatorname{Kom}^{0}(n)$ is well-defined as a power series in $\mathbb{Z}\left[q^{-1}\right] \llbracket q \rrbracket$.

Definition 2.6. The Grothendieck group of Kom is

$$
K_{0}(\mathrm{Kom})=\mathbb{Z}\left\langle\operatorname{Iso}\left(\operatorname{Kom}^{0}\right)\right\rangle /\left(\left[K_{*}\right]=\sum_{i=-\infty}^{\infty}(-1)^{i}\left[K_{i}\right]\right),
$$

the free abelian group generated by isomorphism classes of objects in $\mathrm{Kom}^{0}$ modulo the relation above. We again identify $\left[q^{j} K_{i}\right]=q^{j}\left[K_{i}\right]$, and the monotonicity implies that these relations are well-defined power series. Since the objects of Kom are bounded in negative homological degree the sum above contains only finitely many terms with $i<0$. $K_{0}(\mathrm{Kom})$ inherits an algebra structure as in Definition 2.1. 
Lemma 2.7. There is an isomorphism of $\mathbb{Z}\left[q^{-1}\right] \llbracket q \rrbracket$-algebras

$$
K_{0}(\operatorname{Kom}(n)) \cong \mathrm{TL}_{n}
$$

The proof is standard.

Remark 2.8. The monotonicity condition imposed on $\operatorname{Kom}(n)$ is very natural in our context. Let $f: A \rightarrow B$ be a morphism in $\operatorname{Cob}(n)$, then one of the following holds:

(1) $\operatorname{deg}_{t}(f)>0$ and $f=0$;

(2) $\operatorname{deg}_{t}(f)=0, A=B$, and $f=m 1_{A}$, for some $m \in \mathbb{Z}$;

(3) $\operatorname{deg}_{t}(f)<0$.

This follows from the classification of surfaces and the relations imposed on surfaces in $\operatorname{Cob}(n)$. For instance, the only surface of positive Euler characteristic is the sphere which evaluates to 0 . We conclude that the graded components of nontrivial chain complexes $K_{*}$ in $\operatorname{Kom}(n)$ with degree 0 differential, as in Definition 3.1 below, are monotonically increasing in the sense defined above.

2.4. Chain homotopy lemmas. We will make frequent use of the following standard lemma in this paper; see [4] and [22]. For the definition of homotopy see [10].

Lemma 2.9 (Gaussian elimination). Let $K_{*}$ be a chain complex in an additive category A containing a subcomplex isomorphic to the top row below. If $\varphi: B \rightarrow D$ is an isomorphism there is a homotopy equivalence from $K_{*}$ to a smaller complex containing the bottom row below:

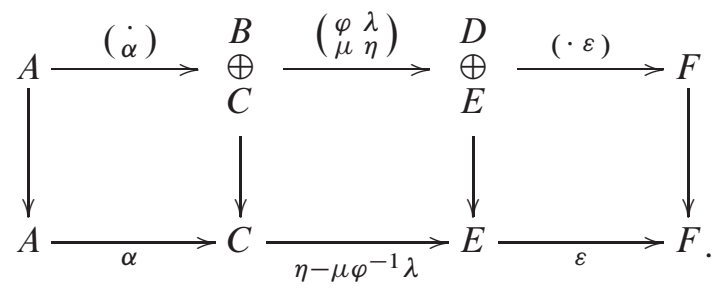

Remark 2.10. Often a preliminary step before an application of Gaussian Elimination will be delooping. This consists of using the isomorphisms $\varphi$ and $\psi$ defined in Section 2.3 to remove a disjoint circle from a diagram and adjust any maps to and from the diagram accordingly. In applications this results in an isomorphism which can be removed using Lemma 2.9. For example, see the proof of Theorem 4.2.

The following result is a direct generalization which will be very useful in our context. 
Lemma 2.11 (simultaneous Gaussian elimination). Let $K_{*}$ be a chain complex in an additive category $\mathcal{A}$ of the form

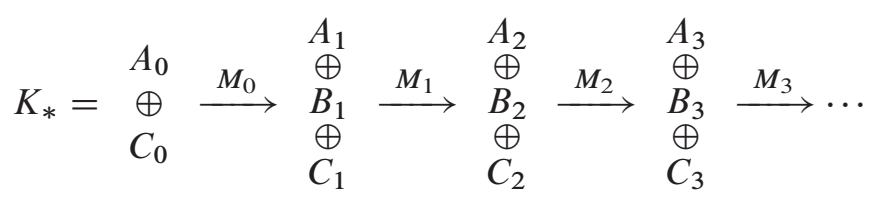

where

$$
M_{0}=\left(\begin{array}{ll}
a_{0} & c_{0} \\
d_{0} & f_{0} \\
g_{0} & j_{0}
\end{array}\right)
$$

and

$$
M_{i}=\left(\begin{array}{ccc}
a_{i} & b_{i} & c_{i} \\
d_{i} & e_{i} & f_{i} \\
g_{i} & h_{i} & j_{i}
\end{array}\right)
$$

for all $i>0$.

If $a_{2 i}: A_{2 i} \rightarrow A_{2 i+1}$ and $e_{2 i+1}: B_{2 i+1} \rightarrow B_{2 i+2}$ are isomorphisms for $i \geq 0$ then the chain complex $K_{*}$ is homotopy equivalent to the smaller chain complex $D_{*}$ obtained by removing all $A_{i}$ and $B_{i}$ terms via the isomorphisms $a_{2 i}$ and $e_{2 i+1}$ :

$$
D_{*}=C_{0} \stackrel{q_{0}}{\longrightarrow} C_{1} \stackrel{q_{1}}{\longrightarrow} C_{2} \stackrel{q_{2}}{\longrightarrow} C_{3} \stackrel{q_{3}}{\longrightarrow} \cdots
$$

where $q_{2 i}=j_{2 i}-g_{2 i} a_{2 i}^{-1} c_{2 i}$ and $q_{2 i+1}=j_{2 i+1}-h_{2 i+1} e_{2 i+1}^{-1} f_{2 i+1}$.

Proof. First apply Gaussian elimination to each isomorphism $a_{2 i}$ in order to obtain the chain complex

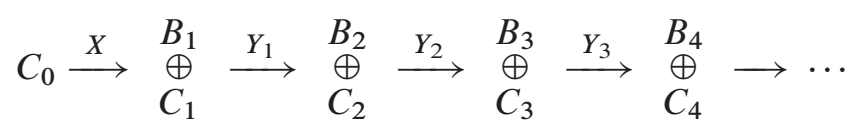

where

$$
X=\left(\begin{array}{c}
f_{0}-c_{0} a_{0}^{-1} d_{0} \\
j_{0}-c_{0} a_{0}^{-1} g_{0}
\end{array}\right)
$$

and

$$
Y_{2 i}=\left(\begin{array}{cc}
e_{2 i}-d_{2 i} a_{2 i}^{-1} b_{2 i} & f_{2 i}-d_{2 i} a_{2 i}^{-1} c_{2 i} \\
h_{2 i}-g_{2 i} a_{2 i}^{-1} b_{2 i} & j_{2 i}-g_{2 i} a_{2 i}^{-1} c_{2 i}
\end{array}\right), \quad Y_{2 i+1}=\left(\begin{array}{ll}
e_{2 i+1} & f_{2 i+1} \\
h_{2 i+1} & j_{2 i+1}
\end{array}\right) .
$$

Now apply Gaussian elimination to each isomorphism $e_{2 i+1}$ in order to obtain the chain complex $D_{*}$ above.

A chain complex $K_{*}$ is contractible if $K_{*} \simeq 0$; see [10].

Lemma 2.12 (big collapse). A chain complex $K_{*}$ of contractible chain complexes $K_{i}$ is contractible. 


\section{Universal projectors and statement of the main theorem}

The projectors defined in this paper satisfy a universal property making them unique up to homotopy.

Definition 3.1. A chain complex $\left(P_{*}, d_{*}\right) \in \operatorname{Kom}(n)$ is a universal projector if the following conditions hold.

(1) The complex is positively graded with degree zero differential:

(a) $P_{k}=0$ for all $k<0$ and $\operatorname{deg}_{q}\left(P_{k}\right) \geq 0$ for all $k>0$;

(b) $d_{k}$ is a matrix of degree zero maps for all $k \in \mathbb{Z}$.

(2) The identity diagram appears only in homological degree zero and only once:

(a) $P_{0} \cong 1$

(b) $P_{k} \nRightarrow 1 \oplus D$ for any $D \in \operatorname{Mat}(\operatorname{Cob}(n))$ and for all $k>0$.

(3) The chain complex $P_{*}$ is contractible "under turnbacks," that is for any generator $e_{i} \in \mathrm{TL}_{n}, 0<i<n$

(a) $P_{*} \otimes e_{i} \simeq 0$ and

(b) $e_{i} \otimes P_{*} \simeq 0$.

See Section 2.3 for a discussion of degrees.

Compare these axioms to the axioms in Section 2.2 characterizing the JonesWenzl projectors $p_{n} \in \mathrm{TL}_{n}$. The first two axioms are non-triviality conditions. The first excludes uninteresting variants of the definition obtained by degree shifting and symmetry. For instance, we could change 3.1(1) to require a negative $q$-grading and reverse of all the arrows. The second excludes contractible complexes from consideration. The third axiom implies that composing the projector with any Temperley-Lieb diagram which is not identity yields an object in $\operatorname{Kom}(n)$ which is homotopic to the zero complex.

If $P_{n} \in \operatorname{Kom}(n)$ is a universal projector then $\left[P_{n}\right] \in K_{0}(\operatorname{Kom}(n)) \cong \mathrm{TL}_{n}$ satisfies the axioms in Section 2.2, implying that $\left[P_{n}\right]=p_{n} \in \mathrm{TL}_{n}$. See Section 2.3.1 for a detailed discussion of what categorification means in this context.

In [18] it was shown that those categorifications of $\mathrm{TL}_{n}$ giving rise to functorial invariants of tangles must be categories containing Frobenius algebra objects which are quotients of the one described by Section 2.3 (see Example 2.2). Since the construction of the universal projector only uses relations which follow from the existence of such an algebra it is possible that the construction of the universal projector in $\operatorname{Kom}(n)$ could be carried out within other categorifications.

It is important to note that our definition disagrees with some previous categorifications based on different axiomatizations of the Jones-Wenzl projectors such as the dimension axiom [17] (for related work see [5] and [11]):

$$
\operatorname{Tr}\left(p_{n}\right)=[n+1] .
$$


This is implied by the homotopy uniqueness corollary below and the computation of $\mathrm{H}_{*}\left(\operatorname{Tr}\left(P_{2}\right)\right)$ contained in the next section.

We can now state the main theorem of the paper.

Theorem 3.2. For each $n>0$, there exists a chain complex $C \in \operatorname{Kom}(n)$ that is a universal projector.

We summarize some immediate consequences of the axioms in Definition 3.1. See also Sections 5 and 6.

Proposition 3.3. If $C \in \operatorname{Kom}(n)$ is a universal projector and $D \in \operatorname{Kom}(m)$ is a universal projector such that $0 \leq m \leq n$ then

$$
C \otimes\left(D \sqcup 1^{n-m}\right) \simeq C \simeq\left(D \sqcup 1^{n-m}\right) \otimes C ;
$$

pictorially

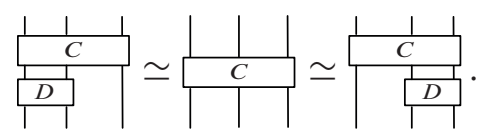

Proof. The tensor product of chain complexes $C_{*} \otimes\left(D_{*} \sqcup 1^{n-m}\right)$ is the total complex of a bicomplex which can be written as a chain complex of chain complexes

$$
C_{*} \otimes\left(D_{0} \sqcup 1^{n-m}\right) \longrightarrow C_{*} \otimes\left(D_{1} \sqcup 1^{n-m}\right) \longrightarrow C_{*} \otimes\left(D_{2} \sqcup 1^{n-m}\right) \longrightarrow \cdots
$$

or, graphically,

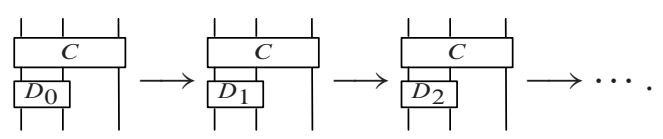

By the second axiom $D_{0}=1$ in homological degree 0 and so the identity diagram cannot be found as a summand of $D_{k} \sqcup 1^{n-m}$ for any $k>1$. In addition, $C$ satisfies 3.1(3) so it follows that

(1) the homological degree 0 portion of this complex is isomorphic to $C_{*}$ and

(2) all chain complexes in degree above zero are contractible.

Lemma 2.12 (big collapse) implies that there is a homotopy equivalence $C_{*} \simeq$ $C_{*} \otimes\left(D_{*} \sqcup 1^{n-m}\right)$. The other equivalence $\left(D_{*} \sqcup 1^{n-m}\right) \otimes C_{*} \simeq C_{*}$ is proven in the same manner.

A special case of the above proposition with $m=n$ implies that universal projectors we have defined behave like idempotent elements. In other words, if $C$ is a universal projector then the functors $C \otimes-$ and $-\otimes C$ are idempotent on the homotopy category of $\operatorname{Kom}(n)$. 
Corollary 3.4 (idempotence). If $C \in \operatorname{Kom}(n)$ is a universal projector then

$$
C \otimes C \simeq C \text {. }
$$

This is represented diagrammatically as

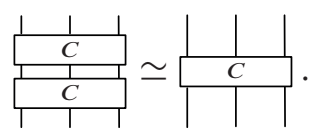

Proposition 3.3 also implies that the universal projectors are unique up to homotopy.

Corollary 3.5 (homotopy uniqueness). If $C, D \in \operatorname{Kom}(n)$ are universal projectors then $C \simeq D$.

Proof. Let $C, D \in \operatorname{Kom}(n)$ be universal projectors. Proposition 3.3 holds when $n=m$ so that $1^{0}=\emptyset$,

$$
C \simeq C \otimes(D \sqcup \emptyset) \cong(C \sqcup \emptyset) \otimes D \simeq D ;
$$

in pictures,

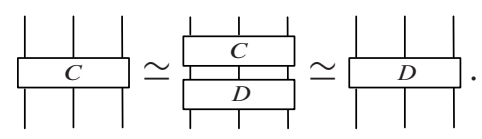

\section{Explicit formulae and computations}

We now give some explicit examples of lower order projectors. The second projector below will play a role in the proof of the main theorem. Higher projectors are much more complicated.

4.1. The second projector. The second projector is defined to be the chain complex

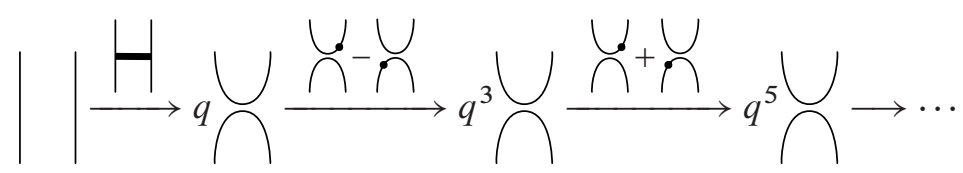

in which the last two maps alternate ad infinitum. More explicitly, we have

$$
P_{2}=\left(C_{*}, d_{*}\right) .
$$

The chain groups are given by

$$
C_{n}=\left\{\begin{array}{l}
q^{0} \mid \\
q^{2 n-1} \bigcup n>0 .
\end{array}\right.
$$


The differential is given by

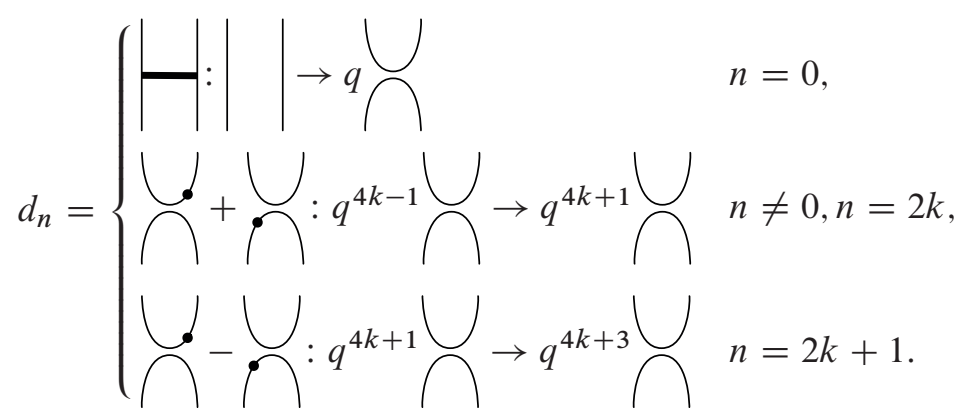

Proposition 4.1. $P_{2}$ defined above is a chain complex, that is successive compositions of the differential are equal to zero.

Proof. Since $d_{2 n+1} \circ d_{2 n}=d_{2 n} \circ d_{2 n-1}$ there are only two cases. We have

$$
\begin{aligned}
d_{1} \circ d_{0} & =|-!| \\
& =!-!|+| \downarrow=0 .
\end{aligned}
$$

The second equality follows from moving the dot from one side of the saddle to the other, and isotopic cobordisms are considered equal by construction, see Section 2.3.

Further we have

$$
\begin{aligned}
& d_{2 n+1} \circ d_{2 n}=(\oiiint+\oiiint) \circ(\oiiint-\wp) \\
& =0+\infty+\infty \\
& =\alpha \circlearrowleft+0-\alpha \circlearrowleft=0 \text {. }
\end{aligned}
$$

The last equality follows because the relations of Section 2.3 allow us to replace two dots by multiplication with $\alpha$.

Theorem 4.2. The chain complex $P_{2} \in \mathrm{Kom}(2)$ defined above is a universal projector.

Proof. Since the identity object only appears in degree 0 and the chain complex is positively graded with degree zero differentials, 3.1(3) and3.1(2) are satisfied by 
definition. For 3.1(3), note that there is only one standard generator $e_{1} \in \mathrm{TL}_{2}$ and the vertical symmetry in the definition of $P_{2}$ implies $P_{2} \otimes e_{1} \cong e_{1} \otimes P_{2}$. Consider $e_{1} \otimes P_{2}$ :

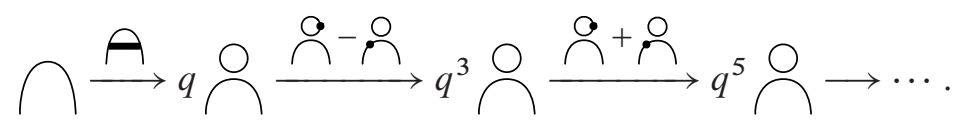

The top strand of $e_{1}$ has been omitted in the illustration above.

Now we "deloop" (see remark in Section 2.10) and conjugate our differentials by the isomorphism $\varphi$ in Section 2.3 to obtain the isomorphic complex

$$
\bigcap \stackrel{M_{0}}{\longrightarrow} q^{0} \bigcap \oplus q^{2} \bigcap \stackrel{M_{1}}{\longrightarrow} q^{2} \bigcap \oplus q^{4} \bigcap \stackrel{M_{2}}{\longrightarrow} q^{4} \bigcap \oplus q^{6} \bigcap \longrightarrow \cdots
$$

where

$$
M_{0}=\left(\begin{array}{l}
\bigcap \\
\bigcap
\end{array}\right), \quad M_{1}=\left(\begin{array}{cc}
-\bigcap & \bigcap \\
\alpha \bigcap & -\bigcap
\end{array}\right), \quad M_{2}=\left(\begin{array}{ll}
\bigcap \bigcap & \bigcap \\
\alpha \bigcap & \bigcap
\end{array}\right) .
$$

Applying Lemma 2.11 (simultaneous Gaussian elimination) using $C_{*}=0$ and selecting for $a_{2 i}$ and $e_{2 i+1}$ the first component of $M_{0}$ and the identity map in the upper right-hand component of each successive matrix shows that the complex is homotopic to the zero complex.

4.1.1. Homology of the trace. In the Temperley-Lieb algebra the trace of any diagram $D \in \mathrm{TL}_{n}$ is defined to be the element $\operatorname{Tr}(D) \in \mathbb{Z}\left[q, q^{-1}\right]$ associated with the diagram obtained by connecting each of the bottom boundary points to the corresponding top points by parallel arcs in the plane:

$$
\operatorname{Tr}(D)=0
$$

The Jones-Wenzl projectors, $p_{n} \in \mathrm{TL}_{n}$ are commonly known to satisfy

$$
\operatorname{Tr}\left(p_{n}\right)=[n+1] .
$$

In fact, they can be characterized by this property together with the turnback 3.1(3).

One would expect then that the graded Euler characteristic of the complex given by the trace of the universal projectors defined in this document are given by the polynomials $[n+1]$. This is true when the coefficient ring is rational and $\alpha=0$. It is however not true that the homology of $\operatorname{Tr}\left(P_{2}\right)$ is spanned only by classes that correspond to coefficients of the graded Euler characteristic; the homology contains infinitely many terms which cancel in the graded Euler characteristic. 
When $\alpha=0$ and coefficients are rational we have

$$
\mathrm{H}_{n}\left(\operatorname{Tr}\left(P_{2}\right) ; \mathbb{Q}\right)= \begin{cases}q^{-2} \mathbb{Q} \oplus q^{0} \mathbb{Q} & n=0, \\ 0 & n=1, \\ q^{4 k-2} \mathbb{Q} & n=2 k \text { and } k>0, \\ q^{4 k+2} \mathbb{Q} & n=2 k+1 \text { and } k>0 .\end{cases}
$$

Note that the graded Euler characteristic equals [3] $=q^{-2}+1+q^{2}$. All other terms cancel in pairs.

If $\alpha=0$ and the coefficient ring is integral then there is an additional infinite family of 2-torsion. If $\alpha \neq 0$ and the coefficient ring is $\mathbb{Q}$ then the homology of $P_{2}$ is 2-dimensional and isomorphic for any choice of $\alpha$. If $\alpha \in \mathbb{Z}_{+}$and the coefficient ring is $\mathbb{Z}$ then there is an infinite family of 2-torsion and an infinite family of $2 \alpha$-torsion. In particular, the homotopy type of the projectors is not constant with respect to the deformation parameter $\alpha$.

Taking the trace of our projector yields a complex with alternating differential

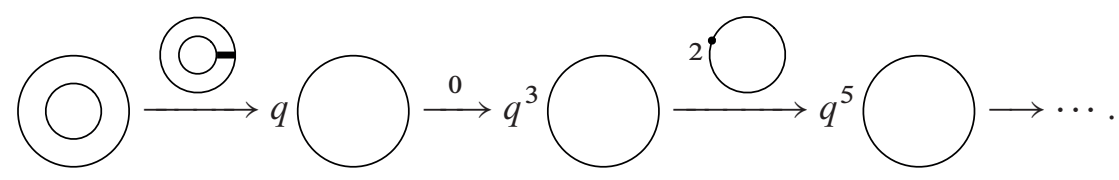

The homology of this complex is in general given by

$$
\mathrm{H}_{n}\left(\operatorname{Tr}\left(P_{2}\right)\right)= \begin{cases}q^{-2} \mathbb{Z} \oplus q^{0} \mathbb{Z} & n=0, \alpha=0 \text { or } \alpha \neq 0, \\ 0 & n=1, \alpha=0 \text { or } \alpha \neq 0, \\ q^{4 k-2} \mathbb{Z} & n=2 k, \alpha=0, \\ q^{4 k+2} \mathbb{Z} \oplus q^{4 k} \mathbb{Z} / 2 & n=2 k+1, \alpha=0, \\ 0 & n=2 k, \alpha \neq 0, \\ q^{4 k+2} \mathbb{Z} /(2 \alpha) \oplus q^{4 k} \mathbb{Z} / 2 & n=2 k+1, \alpha \neq 0 .\end{cases}
$$

4.2. The third projector. We give an inductive definition of the chain complex for the $n$th projector in Section 7 below, with the second projector defined above as the base of the induction. The third projector $P_{3}$ can therefore be deduced from that inductive definition. In this section we present a minimal (in the sense that it is not homotopic to a chain complex containing fewer Temperley-Lieb diagrams) chain complex for $P_{3}$. The third projector is the last which can be written down in a short and explicit diagrammatic form. After the initial identity term the complex below becomes 4 periodic. 
We have

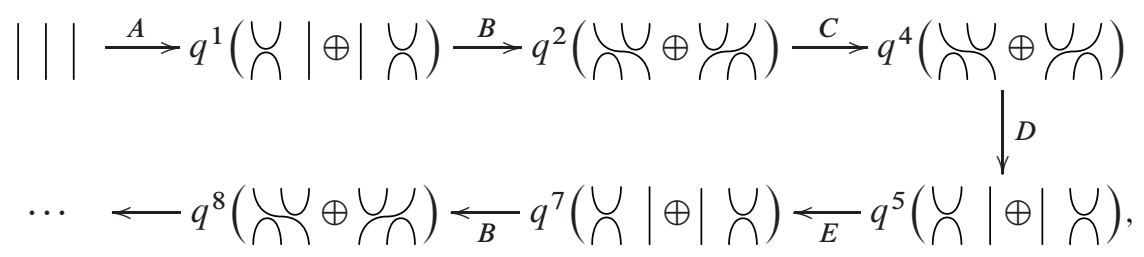

where

$$
\begin{aligned}
& A=(-H|| H)^{\operatorname{tr}}, \\
& B=\left(\begin{array}{cc}
\bigcup & -\Upsilon \bigcap \\
-\bigcup & \bigcup
\end{array}\right) \text {, }
\end{aligned}
$$

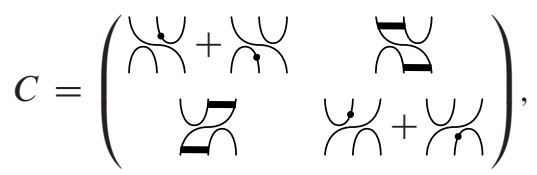

$$
\begin{aligned}
& D=\left(\begin{array}{cc}
\varkappa & -\varkappa \\
-\varkappa & U
\end{array}\right)
\end{aligned}
$$

and

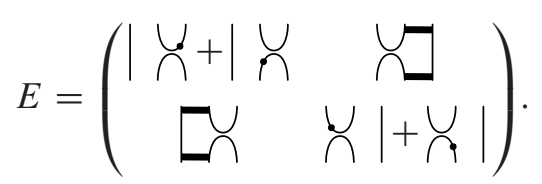

Theorem 4.3. The definition of $P_{3}$ given above is a chain complex that satisfies the axioms of the universal projector. In particular,

$$
e_{i} \otimes P_{3} \simeq 0 \simeq P_{3} \otimes e_{i} \quad i=1,2
$$

The proof is analogous to the proof of Theorem 4.2. The main theorem also produces a universal projector $P_{n}$ for $n=3$.

\section{Reidemeister moves and graphical calculus}

In this section we define invariants of framed tangles obtained by applying the $m$ th projector to the strands of a cabling and showing that the homotopy type of the result is invariant under Reidemeister moves 2 and 3. These are categorifications of the invariants of higher representations of $\mathrm{U}_{q} \mathfrak{s l}(2)$ corresponding to the colored Jones polynomial. 
Definition 5.1. Given $m \in \mathbb{N}$ consider the category $\operatorname{Kom}_{m}(n)$ with objects

$$
\mathrm{Ob}\left(\operatorname{Kom}_{m}(n)\right)=\mathrm{Ob}(\operatorname{Kom}(n)) .
$$

To any object $D \in \mathrm{Ob}(\operatorname{Kom}(n))$ associate a chain complex $F(D)$ in the category $\operatorname{Kom}(m n)$ by replacing each strand in each diagram with $m$ parallel strands composed with the $m$ th projector.

If $A$ and $B \in \operatorname{Kom}_{m}(n)$ are two objects then we define

$$
\operatorname{Hom}_{K_{m}(n)}(A, B)=\operatorname{Hom}_{\mathrm{Kom}(m n)}(F(A), F(B)) .
$$

This can be illustrated by

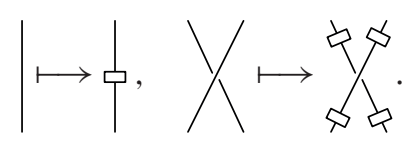

In the remainder of this section we wish to prove that the Reidemeister moves and some standard graphical relations are satisfied up to homotopy.

Lemma 5.2 (projector isotopy). A free strand can be moved over or under a projector up to homotopy. In pictures,

$$
\frac{1}{1} \simeq \frac{1}{\dot{\phi}},-\frac{1}{1} \simeq
$$

Proof. The proof is similar to the proof of Proposition 3.3 and Corollary 3.5 in Section 3. Specifically, observe that both the chain complex for the diagram with the projector below the strand and the chain complex for the diagram with the projector above the strand are chain homotopy equivalent to the chain complex $C$ for the diagram with two projectors: one above the strand and one below the strand. This is true because expanding either of the two projectors in $C$ gives the identity diagram in degree zero and every other term involves a turnback, which is contractible when combined with the second copy of the projector.

Applications of this lemma allow us to show that the Reidemeister moves are satisfied by the category $\operatorname{Kom}_{m}(n)$.

Theorem 5.3. The category $\operatorname{Kom}_{m}(n)$ contains invariants of framed tangles.

Proof. Lemma 5.2 implies that up to homotopy projectors can be slid under or over crossings. Corollary 3.4 implies that up to homotopy any two projectors on the same strand can be replaced with one projector.

For the second Reidemeister move we have

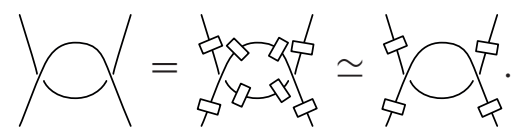


The first equality is by definition. The homotopy equivalence follows from the projector isotopy lemma and $P_{n} \otimes P_{n} \simeq P_{n}$. We have

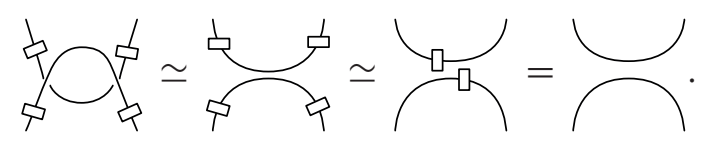

The first homotopy equivalence follows from the second Reidemeister move in the category $\operatorname{Kom}(\mathrm{mn})$; the second follows from another application of $P_{n} \otimes P_{n} \simeq P_{n}$.

The argument for the third Reidemeister move features the same ideas. We have

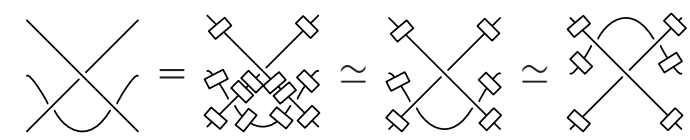

and

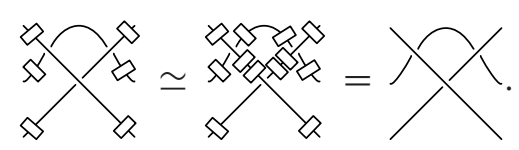

Applying the definition to the standard Reidemeister 3 diagram we obtain a diagram that looks like spaghetti which simplifies considerably up to homotopy to a diagram in which the standard Reidemeister 3 homotopy in $\operatorname{Kom}(m n)$ holds.

Given a framed link $L$ the chain complex associated to $L$ in $\operatorname{Kom}_{n}(0)$ has the graded Euler characteristic of the positive $q$-power series expansion of the $n$th colored Jones polynomial. This is a categorification of the $n$th colored Jones polynomial. See Section 2.3.1 for a detailed discussion of what categorification means in this context.

\section{Spin networks}

In this section we describe how to associate to any spin network a chain complex in a category defined using the universal projector of Section 3. Constructions involving four projectors are then explored more thoroughly leading to a categorification of the $6 j$-symbols.

6.1. Categories and invariants. Let $I=\mathbb{N}$ be the set indexing the finite dimensional irreducible representations of $\mathrm{U}_{q} \mathfrak{s l}(2)$. For any $n$-tuple $\mathbf{t}=\left(i_{1}, \ldots, i_{n}\right) \in I^{n}$ define the invariants of the $n$-fold tensor product by

$$
\operatorname{Inv}(\mathbf{t})=\operatorname{Inv}\left(V_{i_{1}} \otimes \cdots \otimes V_{i_{n}}\right)=\operatorname{Hom}_{\mathrm{U}_{q} \mathfrak{s l}(2)}\left(V_{i_{1}} \otimes \cdots \otimes V_{i_{n}}, 1\right) .
$$

This space is described by Temperley-Lieb diagrams or unoriented isotopy classes of 1-manifolds in a disk with boundary fixed in the boundary of the disk and with boundary labeled by Jones-Wenzl projectors: $p_{i_{1}} \sqcup p_{i_{2}} \sqcup \cdots \sqcup p_{i_{n}}$. See the illustration below or also [14] and [20]. 
For any such $\mathbf{t} \in I^{n}$ the main theorem allows us to construct a category $\operatorname{Kom}(\mathbf{t})$ with objects given by chain complexes obtained from Temperley-Lieb diagrams with boundary labeled by universal projectors and morphisms given by chain maps. When $\mathbf{t}=(a, b, c, d)$ there is an associated picture

$$
\mathrm{Ob}(\operatorname{Kom}(\mathbf{t}))=\{\overbrace{D}: D \text { is a Temperley-Lieb diagram }\} .
$$

The axiomatic correspondence between the Jones-Wenzl projector and the universal projectors in this paper implies the following theorem.

Theorem 6.1. The category $\operatorname{Kom}(\mathbf{t})$ categorifies the invariants $\operatorname{Inv}(\mathbf{t})$.

See Section 2.3.1 for a detailed discussion of what categorification means in this context.

6.2. $\mathbf{6 j}$-symbols. There is a standard way to resolve (compare [14], Chapter 4.1) a trivalent vertex with edges labeled by $a, b, c \in I$ in a spin network:

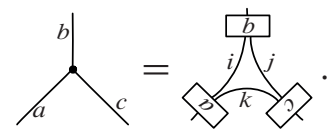

where $i=(a+b-c) / 2, j=(a+c-b) / 2$ and $k=(b+c-a) / 2$ are the number of unoriented intervals placed between the projectors. We say that a diagram is admissible if all trivalent vertices can be resolved using the assigned labels or equivalently $a+b+c$ is even and the triangle inequalities hold for $a, b$ and $c$. Using this notation we can describe two bases for $\operatorname{Inv}(a, b, c, d)$,

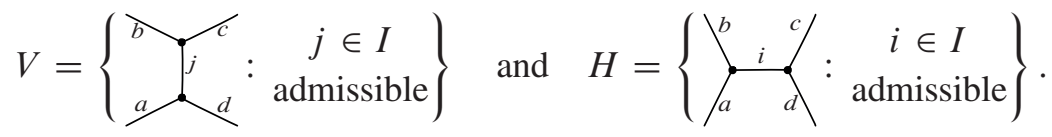

The base change coefficients are called $6 j$-symbols,

$$
\left.\sum_{a}^{b} i_{d}^{c}=\sum_{j}\left\{\begin{array}{lll}
a & b & i \\
c & d & j
\end{array}\right\}_{a}^{b}\right]_{d}^{c},
$$

which determine the change of basis map $S: H \rightarrow V . S$ is the matrix of $6 j$-symbols,

$$
S_{i j}=\left\{\begin{array}{lll}
a & b & i \\
c & d & j
\end{array}\right\} .
$$


Outline. Our goal is to categorify $S$ as a functor $\operatorname{Kom}(a, b, c, d) \rightarrow \operatorname{Kom}(a, b, c, d)$. Our construction is modeled on the linear-algebraic proof (see [14]; Chapter 7.2) that the "vertical" and "horizontal" collections $V, H$ above are indeed bases for the space of Temperley-Lieb diagrams $\operatorname{Inv}(a, b, c, d)$, pictured on the previous page. The key point is that the identity diagram appears only once in the chain complex for the projector $P_{n}$, in degree zero (Definition 3.1(1) of the universal projector). Therefore, the identity diagram may be represented up to homotopy as the cone of the inclusion of the positive degree part into the chain complex $P_{n}$. However the positive degree part may in turn be inductively represented as an iterated cone on lower order projectors. This is made precise in the proof of Theorem 6.4 below. We begin by introducing a categorical analogue of a linear basis.

Before proceeding we recall a number of definitions. The concept we wish to capture is that of a category which is homotopy equivalent to some subcategory sitting inside of it. In our case this amounts to a category of complexes in which every chain complex is homotopy equivalent to a chain complex of chain complexes contained within the subcategory of interest. All of the categories involved in our discussion will be categories of chain complexes of direct sums of objects in a small additive category.

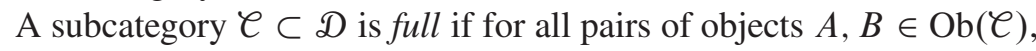

$$
\operatorname{Hom}_{\mathcal{C}}(A, B)=\operatorname{Hom}_{\mathcal{D}}(A, B) \text {. }
$$

A category $\mathcal{C}$ is differential graded if for all objects $A, B \in \mathrm{Ob}(\mathcal{C})$, Hom $\operatorname{Co}(A, B)$ is a chain complex. A functor of differential graded categories $F: \mathcal{C} \rightarrow \mathscr{D}$ is a differential graded functor if the maps

$$
F_{A, B}: \operatorname{Hom}_{\mathcal{C}}(A, B) \longrightarrow \operatorname{Hom}_{\mathfrak{D}}(F(A), F(B))
$$

are chain maps for all objects $A, B \in \mathcal{C}$. Two differential graded functors $F, G: \mathcal{C} \rightarrow$ $D$ are homotopic, $F \simeq G$ if there is a natural transformation $\varphi: F \rightarrow G$ such that $\varphi_{A}$ is a homotopy equivalence for all $A \in \mathrm{Ob}(\mathcal{C})$. Two differential graded categories $\mathcal{C}$ and $\mathscr{D}$ are homotopy equivalent if there exist differential graded functors $F: \mathcal{C} \rightarrow \mathscr{D}$ and $G: D \rightarrow \mathcal{D}$ such that $F G \simeq 1_{\mathscr{D}}$ and $G F \simeq 1_{\mathcal{C}}$.

Definition 6.2. If $\mathcal{A}$ is an additive category and $\mathscr{C}=\operatorname{Kom}(\mathcal{A})$ is the category of chain complexes of finite direct sums of objects in $\mathcal{A}$ then a full subcategory $B \subset \mathcal{C}$ spans $\mathcal{C}$ if the inclusion $\mathscr{B} \hookrightarrow \mathcal{C}$ is a homotopy equivalence of categories.

Lemma 6.3. For any $a, b, c, d \in \mathbb{N}$ the category $\operatorname{Kom}(a, b, c, d)$ is naturally spanned by the full subcategory $\mathcal{N}$ :

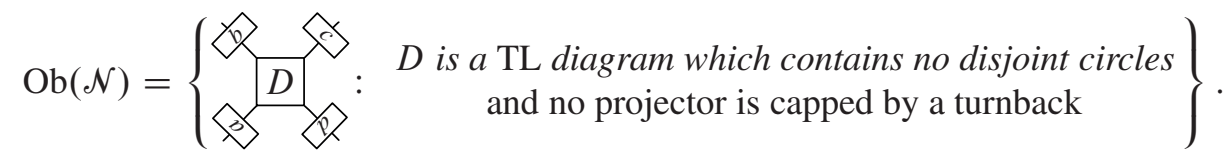


Proof. Axioms 3.1(3a) and 3.1(3b) imply that projectors with turnbacks are contractible. Using the isomorphisms of Section 2.3 any complex associated to a diagram with disjoint circles is isomorphic to a finite direct sum of chain complexes associated to diagrams without circles. This defines a functor

$$
\pi: \operatorname{Kom}(a, b, c, d) \longrightarrow \mathcal{N}
$$

which together with the obvious inclusion functor

$$
i: \mathcal{N} \hookrightarrow \operatorname{Kom}(a, b, c, d)
$$

satisfy

$$
\pi i=1_{\mathcal{N}} \quad \text { and } \quad i \pi \simeq 1_{\mathrm{Kom}(a, b, c, d)} .
$$

There are two other categories we would like to consider: $\mathscr{H}$ and $\mathcal{V}$. These are the full subcategories of $\operatorname{Kom}(a, b, c, d)$ with objects given by horizontal and vertical diagrams respectively.

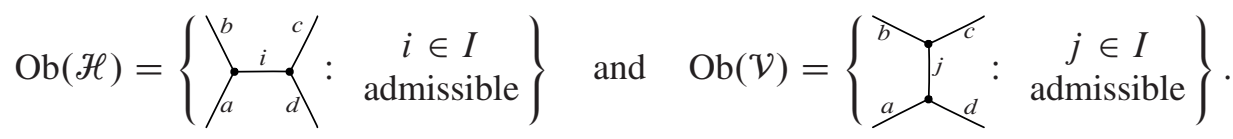

We can now state our theorem.

Theorem 6.4. For any $a, b, c, d \in I$ the full subcategories $\mathscr{H}$ and $\mathcal{V}$ defined above span the category $\operatorname{Kom}(a, b, c, d)$.

The proof consists of constructing a family of chain complexes $V_{n}$ and $H_{n}$ each of which comes from the positive degree part of the chain complex defining the $n$th universal projector $P_{n}$ constructed above. The gist of the proof is captured by the two tables below in which $(a, b, c, d)=(2,2,2,2)$ :

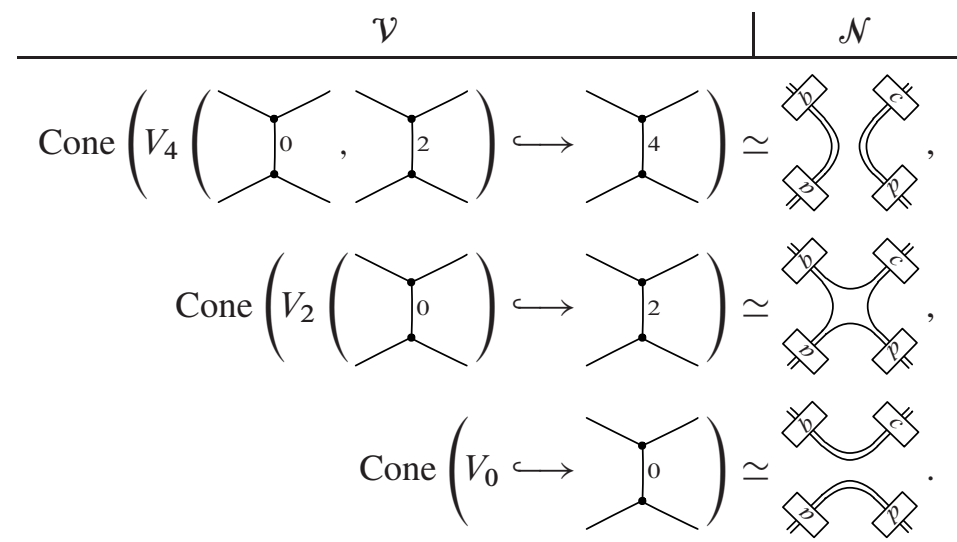

and 


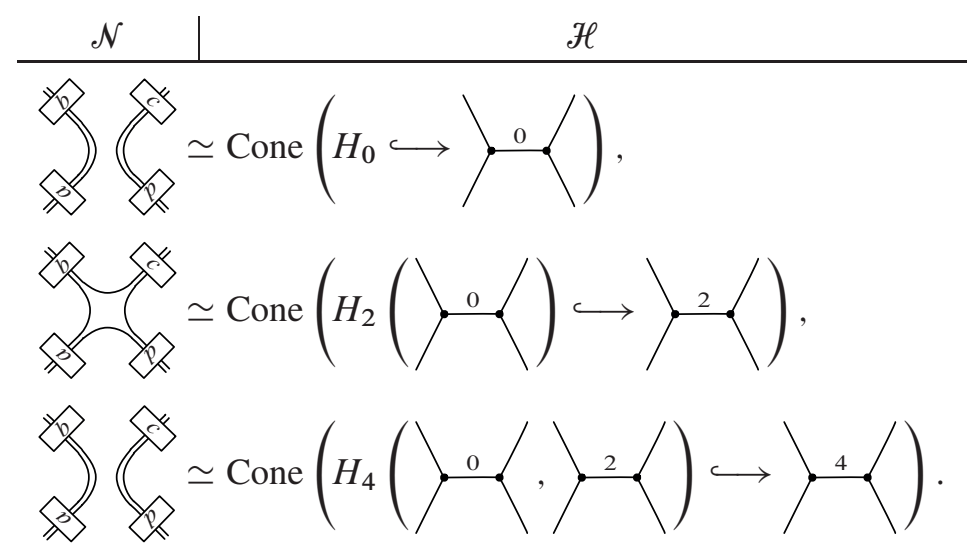

In the first table, the third chain complex

$$
x=
$$

can be constructed as a cone on the vertical spin network

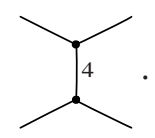

When $(a, b, c, d)=(2,2,2,2)$ and $V_{0}=0$, the spin network itself is equal to $X$. Next, to define the chain complex $V_{2}$ expand the central projector of

$$
Y=r_{2}
$$

and then substitute instances of $X$ with

$$
\text { Cone }\left(V_{0} \longleftrightarrow 0_{0}\right) \text {. }
$$

The chain complex $V_{2}$ consists only of the resulting terms with the 0 labeled edge. The cone on the inclusion of $V_{2}$ into $Y$ is homotopic to

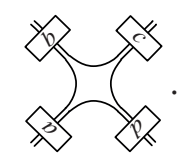

Finally, the first line states that the object

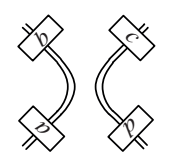


is homotopic to the cone on a chain complex $V_{4}$ consisting of only vertical networks labeled 0 and 2. We now give a proof of Theorem 6.4.

Proof. We will explain the construction only for $\mathcal{V}$ since the argument is the same for $\mathscr{H}$. For fixed $a, b, c, d \in I$ each diagram $D$ defining an object in the spanning subcategory $\mathcal{N}$ defined above must be of the form

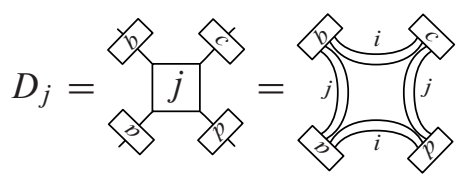

where $j$ is the total number of vertical strands and $i$ is the total number of horizontal strands. Notice $i$ depends on $j$ and if the diagram is admissible the number $j$ assumes either odd or even integer values between two numbers $l_{0}, l_{N} \in \mathbb{Z}_{+}$. If we use $D_{n}$ to denote the diagram with $j=n$ in the illustration above then for a given $a, b, c, d \in I$ we have diagrams $D_{l_{0}}, D_{l_{0}+2}, \ldots, D_{l_{N}-2}, D_{l_{N}}$. The proof is by induction on the number of vertical strands.

For each $a, b, c, d \in I$ and admissible $j$ we define the chain complex $V_{j}$,

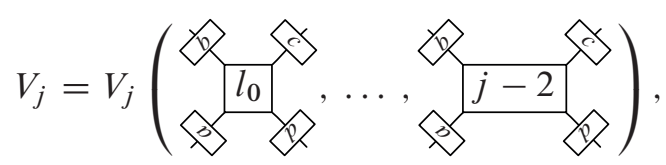

to be the subcomplex

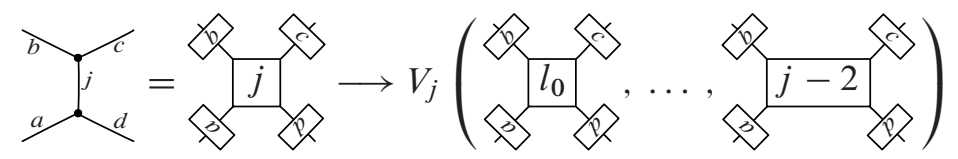

obtained by expanding the central projector $P_{j}$. As our notation suggests $V_{j}$ is a chain complex containing only contractible terms and objects of $\mathcal{N}$ defined by diagrams $D_{l_{0}}, \ldots, D_{j-2}$.

The preceding diagram implies that the homotopy equivalence below is tautological:

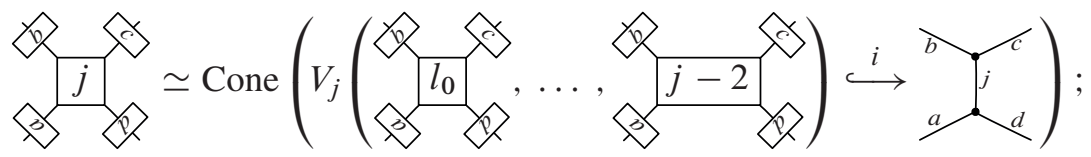

where $i$ is the inclusion of the subcomplex $V_{j}$ into the complex representing the labeled graph. This inclusion exists by construction of $V_{j}$ above. The diagrams appearing in $D_{0}, \ldots, D_{j-2}$ have fewer vertical strands and are homotopic to diagrams containing only vertical spin networks by induction:

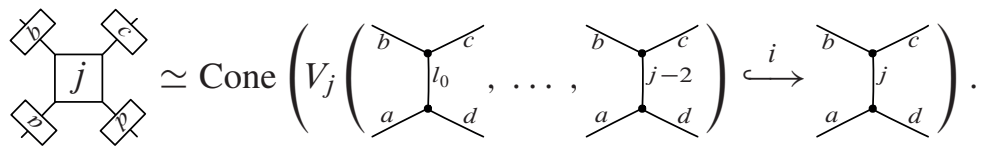


Again $i$ is the inclusion of the subcomplex $V_{j}$ into the complex representing the labeled graph. The proof that substitution works is an application of the change of basis isomorphism used in the Gaussian elimination lemma in Section 2.4 to the first vertical identity map below:

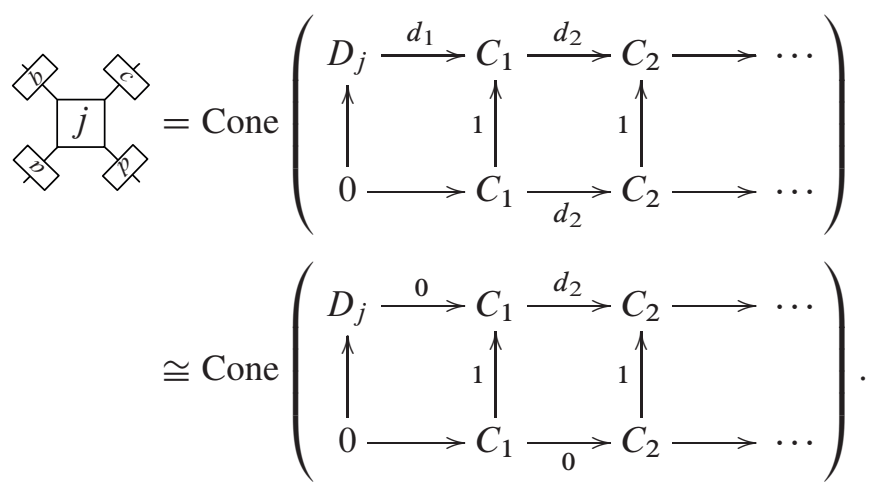

Note that in the base case, expanding the projector $p_{l_{0}}$ yields only contractible terms in degree greater than zero. The $a, b, c, d, l_{0}$ labeled network is homotopy equivalent to the cone on a nullhomotopic map of the form above.

Remark 6.5. The naturally defined homotopy equivalence of categories $S: \mathscr{H} \rightarrow \mathcal{V}$ may be viewed as a categorical analogue of the matrix of 6j symbols $S: H \rightarrow V$ defined in Section 6.2. See Section 2.3.1 for further discussion of what categorification means in this context.

The quantum reader is invited to prove the homotopy Biedenharn-Elliot identity.

\section{Proof of the main theorem}

The two term recurrence relation satisfied by the Jones-Wenzl projectors in Section 2.2 is quadratic in the sense that in order to define $p_{n}$ the $(n-1)$-st projector $p_{n-1}$ appears twice in the second term. One obtains the linear recurrence of Frenkel and Khovanov [7] by expanding the bottom $p_{n-1}$ term completely and removing terms containing a turnback $p_{n-1} e_{i}$ for any $0<i<n-1$. Keeping track of the coefficients in this process gives the recurrence

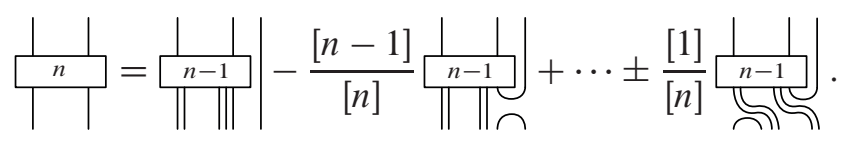

Note that our sign conventions differ from [7]. This can be shown to satisfy 3.1(1-3) in Section 2.2 and so is equal to the Jones-Wenzl projector. In this section we prove the 
main theorem of the paper by constructing a chain complex in the category $\operatorname{Kom}(n)$, motivated by the Frenkel-Khovanov recursive formula above, satisfying the axioms of the universal projector given in Section 3.

7.1. Triples and quadruples. We will begin by examining some situations in which local cancellations can be made in a chain complex containing a turnback: $C_{*} \otimes e_{i}$. There are two important cases: either a sequence of three terms can be canceled after delooping the middle term or a sequence of four terms can be canceled after delooping the two middle terms. We will call the first case a triple and the second a quadruple. Both cases are necessary to prove $P_{3} \otimes e_{i} \simeq 0$ and, as we will see, they suffice to prove the general case.

\subsubsection{The triple}

Definition 7.1. If $D \in \operatorname{Kom}(n)$ is any chain complex and $e_{i}$ is a standard generator of $\mathrm{TL}_{n}$ then an $i$-triple or triple is a sequence of maps in $\operatorname{Kom}(n)$ of the form

$$
D \longrightarrow q D \otimes e_{i} \longrightarrow q^{2} D \otimes e_{i} \otimes e_{i \pm 1},
$$

where the maps are given by saddles, as illustrated below:

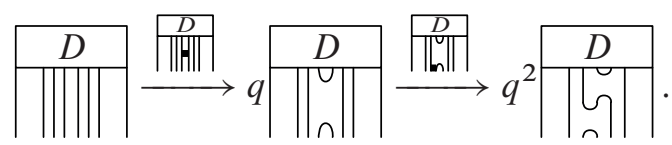

Applying the functor $-\otimes e_{i}$ to the above yields the top sequence in the following commutative diagram:

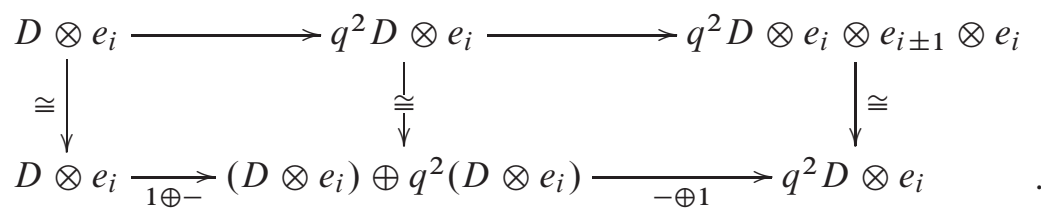

After applying $-\otimes e_{i}$ to an $i$-triple, the middle term can be delooped (see Remark 2.10) yielding the second isomorphism. The last term satisfies the categorified planar isotopy relation 2 of Section 2, yielding the bottom sequence in the diagram above. Note that if this triple is part of a chain complex, then it can be canceled using two applications of the Gaussian elimination (Lemma 2.9 in Section 2.4) or by a single application of the simultaneous Gaussian Elimination (Lemma 2).

\subsubsection{The quadruple}

Definition 7.2. If $D \in \operatorname{Kom}(n)$ is any chain complex and $e_{i}$ an elementary generator of $\mathrm{TL}_{n}$ then an $i$-quadruple or quadruple is a sequence of maps in $\operatorname{Kom}(n)$ of the form

$$
D \longrightarrow q D \otimes e_{i} \stackrel{A}{\longrightarrow} q^{3} D \otimes e_{i} \longrightarrow q^{4} D \otimes e_{i} \otimes e_{i \pm 1} .
$$


All maps are given by saddles except for the $q$-degree 2 map $A$,

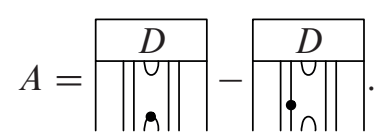

$A$ is given by subtracting the addition of a handle on one strand from the addition of a handle on an adjacent strand. Although this will not affect the arguments below, we will fix the convention that the dot is placed either to the left or the right of the saddle in the second term, depending upon whether the $e_{i+1}$ or $e_{i-1}$ saddle is used at the end of the quadruple. The entire sequence of maps can be pictured as

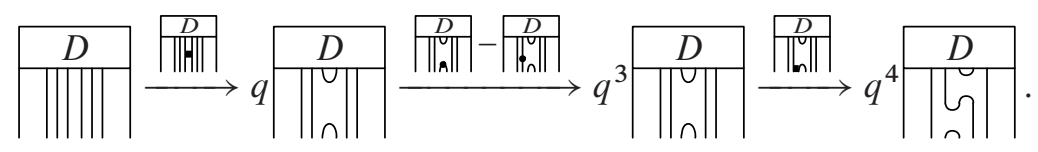

Applying the functor $-\otimes e_{i}$ yields the top row of the following diagram:

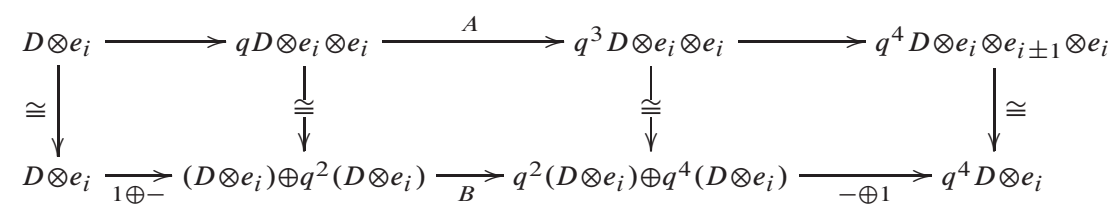

in which $B$ is of the form

$$
B=\left(\begin{array}{c}
-1 \\
--
\end{array}\right) .
$$

Compare to delooping in Section 4.1 and Remark 2.10.

Again note that if this quadruple is part of a chain complex, then it can be canceled using three applications of the Gaussian elimination (or a single application of the simultaneous Gaussian Elimination).

7.2. The Frenkel-Khovanov sequence. The Frenkel-Khovanov formula from the beginning of this section suggests the following recursive definition: for $n=1$

$$
\begin{aligned}
& 1 \\
& \hline 1
\end{aligned}=\mid
$$

for $n=2$

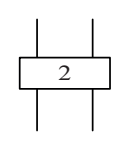

is defined in Section 4.1; and for $n>2$

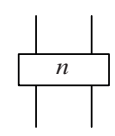


is defined as
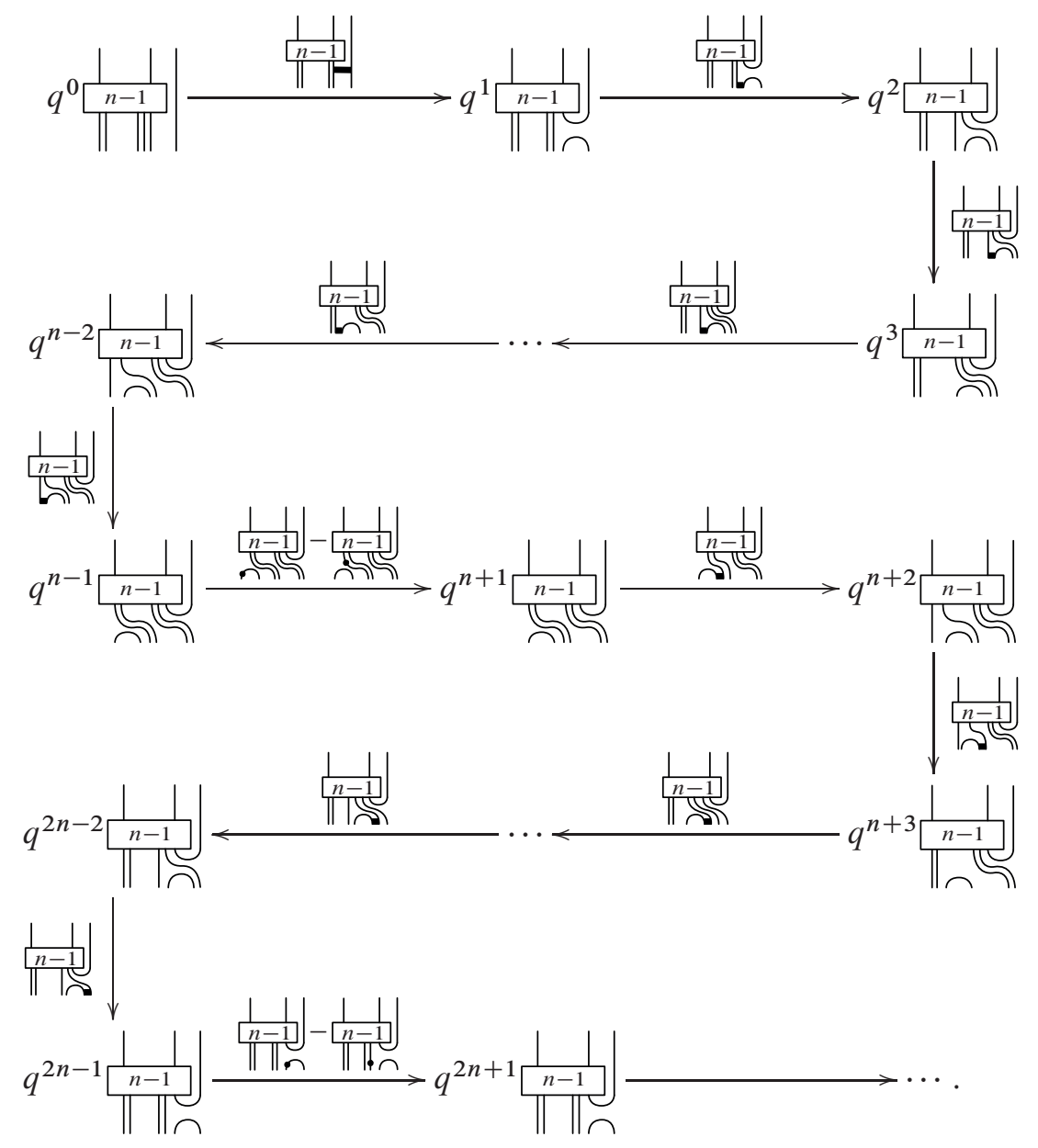

Although the proposition below implies that this definition behaves "correctly" with respect to the turnback axiom 3.1(3) of the universal projector (see Section 3), the composition of two saddles is not equal to zero (although it is homotopic to zero). The technical heart of this paper consists of a detour taken purely for the purpose of arriving at an actual chain complex. The final formulation obtained in Section 7.3 will amount to a version of the above which has been carefully thickened by contractible summands. See Definition 7.9 and the picture following it. The reader is encouraged to check that the graded Euler characteristic of the sequence illustrated above is a formal power series corresponding to the Frenkel-Khovanov recursion formula for $p_{n} \in \mathrm{TL}_{n}$.

In order to formalize the definition above consider the category $\mathbb{N}$ determined by 
the graph

$$
0 \stackrel{d^{0}}{\longrightarrow} 1 \stackrel{d^{1}}{\longrightarrow} 2 \stackrel{d^{2}}{\longrightarrow} \cdots .
$$

The objects of $\mathbb{N}$ are non-negative integers and the morphisms are freely generated by compositions of identity morphisms $1_{i}: i \rightarrow i$ and morphisms $d^{i}: i \rightarrow i+1$.

Definition 7.3. A sequence $F$ in $\operatorname{Kom}(n)$ is a functor

$$
F: \mathbb{N} \longrightarrow \operatorname{Kom}(n) .
$$

For each $n \geq 1$, we will define a sequence $\mathrm{FK}_{n}: \mathbb{N} \rightarrow \operatorname{Kom}(n)$. $\mathrm{FK}_{n}(k)$ will correspond to the bottom of each diagram in the illustration on the previous page (the illustration itself equals $\left.\left(P_{n-1} \sqcup 1\right) \otimes \mathrm{FK}_{n}\right)$. After the initial identity diagram, $\mathrm{FK}_{n}$ is $2(n-1)$ periodic. The following is an algebraic definition of the diagrams pictured above.

Definition 7.4. If $m \in \mathbb{Z}_{+}$write $m=2(n-1) q+r$ with $0<r \leq 2(n-1)$ then the $m$ th diagram of the $n$th Frenkel-Khovanov sequence is defined by

$$
\mathrm{FK}_{n}(m)= \begin{cases}1 & \text { if } m=0, \\ q^{m} e_{n-1} \otimes \ldots \otimes e_{n-m} & \text { if } 1 \leq m<n, \\ q^{2(m-n+1)} \mathrm{FK}_{n}(2 n-m-1) & \text { if } n \leq m \leq 2(n-1), \\ q^{2 n} \mathrm{FK}_{n}(r) & \text { otherwise. }\end{cases}
$$

We use the multiplicativity of the formal $q$-grading: $q^{i}\left(q^{j} D\right)=q^{i+j} D$ for any $D \in \operatorname{Kom}(n)$. The differential between any two objects whose $q$-degree differs by one is given by a saddle map. In each period the two $q$-degree 2 differentials are defined to be those illustrated in the diagram above. (The degree 2 maps in the sequence are separated by $n-2$ saddle maps). In what follows $f_{m}$ will be used to denote the differential, $f_{m}: \mathrm{FK}_{n}(m) \longrightarrow \mathrm{FK}_{n}(m+1)$.

For a given length $l \geq 0$ the $n$th truncated Frenkel-Khovanov sequence is given by

$$
\mathrm{FK}_{n, l}(m)= \begin{cases}\mathrm{FK}_{n}(m) & \text { if } m \leq l \\ 0 & \text { otherwise }\end{cases}
$$

The following proposition and its corollary are key ingredients in the proof of contractibility under turnbacks contained in Section 7.3.

Proposition 7.5. Let $\mathrm{FK}_{n}: \mathbb{N} \rightarrow \operatorname{Kom}(n)$ be the nth Frenkel-Khovanov sequence defined above. Then for any standard generator $e_{i}, i=1, \ldots, n-1$,

$$
\mathrm{FK}_{n}(-) \otimes e_{i}: \mathbb{N} \longrightarrow \operatorname{Kom}(n)
$$


is a sequence such that for every $k \in \mathbb{N}$ the diagram $\operatorname{FK}_{n}(k) \otimes e_{i} \in \operatorname{Kom}(n)$ either

(1) satisfies a commutativity condition: there exists a Temperley-Lieb element $D \in \operatorname{Cob}(n)$ such that

$$
\mathrm{FK}_{n}(k) \otimes e_{i} \cong e_{j} \otimes D,
$$

where $j=i, i-1$ or $i-2$, or

(2) is contained in an $i$-triple or $i$-quadruple sequence.

The case (1) above when $j=i$ can be pictured by

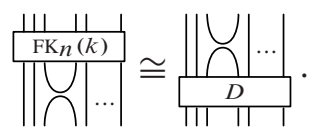

Before giving the proof, we note that once $\mathrm{FK}_{n}$ is part of the chain complex for the universal projector (defined further below), both conclusions (1) and (2) above imply that all terms in $\left(P_{n-1} \sqcup 1\right) \otimes \mathrm{FK}_{n} \otimes e_{i}$ may be contracted. In the case (1) this will follow by the inductive contractibility of $P_{n-1}$ under turnbacks. The contractibility in case (2) follows from the analysis of triples and quadruples in Section 7.1.

Proof. The periodicity of the diagram $\mathrm{FK}_{n}$ ensures that inspecting the first $2 n+1$ terms of $\mathrm{FK}_{n} \otimes e_{i}$ is sufficient. Geometrically inclined readers are invited to prove the proposition by examining the illustration at the beginning of this section. Expanding $\mathrm{FK}_{n}$ allows us to write the first period of $\mathrm{FK}_{n} \otimes e_{i}$ as follows:

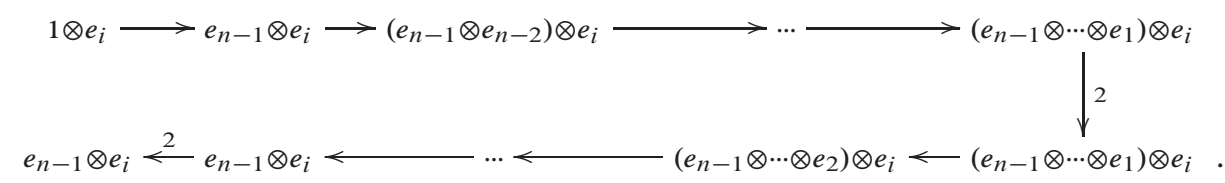

We have dropped the $q$-grading because it is implied by the requirement that the first axiom (in Definition 3.1) holds. We write " 2 " above arrows in order to indicate which maps are of $q$-degree 2 and all other maps are given by saddles.

There are several cases to consider. The first two are boundary cases $i=1$ and $i=n-1$ and the last is the generic case for $1<i<n-1$.

(1) If $i=1$ then consider $-\otimes e_{1}$. If $n-k>2$ then because of the far commutativity relation

$$
\left(e_{n-1} \otimes e_{n-2} \otimes \cdots \otimes e_{n-k}\right) \otimes e_{1} \cong e_{1} \otimes\left(e_{n-1} \otimes e_{n-2} \otimes \cdots \otimes e_{n-k}\right),
$$

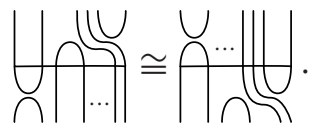


The terms corresponding to $n-k=1$ and $n-k=2$ fit in the following four term sequence:

$$
\alpha \otimes e_{1} \longrightarrow \alpha \otimes e_{1} \otimes e_{1} \stackrel{2}{\longrightarrow} \alpha \otimes e_{1} \otimes e_{1} \longrightarrow \alpha \otimes e_{1},
$$

where $\alpha=e_{n-1} \otimes e_{n-2} \otimes \cdots \otimes e_{2}$. This sequence forms a 1-quadruple (see Section 7.1.2).

(2) If $i=n-1$ then when $k>2$ we have

$$
\left(e_{n-1} \otimes e_{n-2} \otimes \cdots \otimes e_{n-k}\right) \otimes e_{n-1} \cong e_{n-3} \otimes\left(e_{n-1} \otimes e_{n-2} \otimes \cdots \otimes e_{n-k}\right),
$$

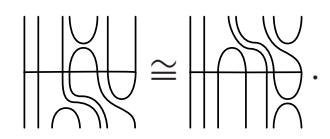

When $k \leq 2$, the first three terms form an $(n-1)$-triple (see Section 7.1.1)

$$
1 \otimes e_{n-1} \longrightarrow e_{n-1} \otimes e_{n-1} \longrightarrow e_{n-1} \otimes e_{n-2} \otimes e_{n-1} \text {. }
$$

After the first period there is an $(n-1)$-quadruple surrounding every other degree 2 map:

$e_{n-1} \otimes e_{n-2} \otimes e_{n-1} \longrightarrow e_{n-1} \otimes e_{n-1} \stackrel{2}{\longrightarrow} e_{n-1} \otimes e_{n-1} \longrightarrow e_{n-1} \otimes e_{n-2} \otimes e_{n-1}$

(3) If $i \neq 1$ and $i \neq n-1$, each term has the form

$$
\left(e_{n-1} \otimes e_{n-2} \otimes \cdots \otimes e_{n-k}\right) \otimes e_{i}
$$

for some $k$ such that $2 \leq k<n-1$. Depending on $k$ there are several cases to consider.

(a) If $n-k>i+1$ then $e_{i}$ commutes with $e_{j}$ for all $j, n-k \leq j \leq n-1$ because of the far commutativity relation. It follows that

$$
\left(e_{n-1} \otimes e_{n-2} \otimes \cdots \otimes e_{n-k}\right) \otimes e_{i} \cong e_{i} \otimes\left(e_{n-1} \otimes e_{n-2} \otimes \cdots \otimes e_{n-k}\right) .
$$

This can be pictured in the same way as the other application of the far commutativity relation in (1).

(b) If $n-k<i-1$, then, similarly,

$$
\left(e_{n-1} \otimes e_{n-2} \otimes \cdots \otimes e_{n-k}\right) \otimes e_{i} \cong e_{i-2} \otimes\left(e_{n-1} \otimes e_{n-2} \otimes \cdots \otimes e_{n-k}\right),
$$

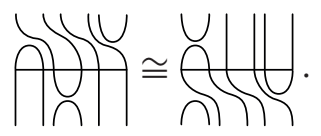


(c) The terms in which $n-k=i-1, n-k=i$ and $n-k=i+1$ form an $i$-triple. For instance,

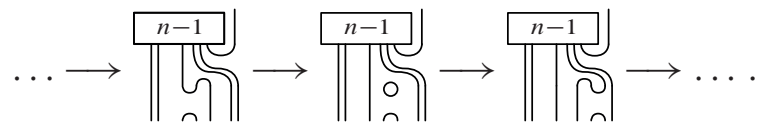

Let $f_{m}$ be the $m$ th map in the Frenkel-Khovanov sequence,

$$
f_{m}: \mathrm{FK}_{n}(m) \longrightarrow \mathrm{FK}_{n}(m+1)
$$

(see Definition 7.4). Recall that each map $f_{m}$ has $q$-degree equal to either 1 or 2 . All degree 1 maps are given by saddle cobordisms and the degree 2 maps are shown in the diagram at the beginning of Section 7.2.

Corollary 7.6. Suppose $P_{n-1} \in \operatorname{Kom}(n-1)$ is an $(n-1)$-st universal projector and let $l \in \mathbb{N}$ be such that $f_{l}$ is a degree 1 map. Then each term in the truncated sequence

$$
f_{l}\left(\left(P_{n-1} \sqcup 1\right) \otimes \mathrm{FK}_{n, l-1}\right)
$$

either is the projector $P_{n-1}$ capped with a turnback or is contained in a triple or a quadruple.

Proof. The proof follows from Proposition 7.5 since the map $f_{l}$ is assumed to be a degree 1 map, that is a saddle cobordism. Therefore the sequence $f_{l}\left(\left(P_{n-1} \sqcup 1\right) \otimes\right.$ $\left.\mathrm{FK}_{n, l}\right)$ equals $\left(\left(P_{n-1} \sqcup 1\right) \otimes \mathrm{FK}_{n, l}\right) \otimes e_{i}$ for some $i$.

The point of this corollary is that if $f_{l}\left(\left(P_{n-1} \sqcup 1\right) \otimes \mathrm{FK}_{n, l}\right)$ is part of a chain complex, then it can be contracted. (In the first case, the projector $P_{n-1}$ capped with a turnback is contractible by Axiom 3.1(1) of the universal projector $P_{n-1}$. In the second case, each triple or quadruple is contractible according to the analysis in Sections 7.1.1 and 7.1.2). This will play an important role in the proof of the main theorem below.

7.2.1. The homotopy projector. If an $(n-1)$-st universal projector $P_{n-1}$ exists then Corollary 7.6 shows that the Frenkel-Khovanov sequence can be used to define a sequence which satisfies the axioms for an $n$th universal projector (Definition 3.1) up to homotopy.

Definition 7.7. The $n$th homotopy projector $\mathrm{HP}_{n}: \mathbb{N} \rightarrow \operatorname{Kom}(n)$ is the sequence defined by

$$
\begin{aligned}
& \mathrm{HP}_{1}=1, \\
& \mathrm{HP}_{n}=\operatorname{Tot}\left(\left(P_{n-1} \sqcup 1\right) \otimes \mathrm{FK}_{n}\right) .
\end{aligned}
$$


For a given length $l \geq 0$ the truncated homotopy projector is defined using the truncated Frenkel-Khovanov sequence

$$
\mathrm{HP}_{n, l}=\left(P_{n-1} \sqcup 1\right) \otimes \mathrm{FK}_{n, l} .
$$

Remark 7.8. A picture of $\mathrm{HP}_{n}$ is given at the beginning of this section. For each $e_{i}, 0<i<n$ by the above proposition $\operatorname{HP}_{n}(k) \otimes e_{i}$ is either a term containing $\left(\mathrm{HP}_{n-1} \otimes e_{j}\right) \sqcup 1$ where $0<j<n-1$ or fits into an $i$-triple or $i$-quadruple. If this were a chain complex then it would be contractible by the lemmas of Section 2.4.

7.3. Construction of the chain complex: fattening the FK sequence. The remark at the end of Section 7.2.1 implies that the sequence $\mathrm{HP}_{n}$ (Definition 7.7) behaves like a universal projector. However it is not a chain complex: the composition of any two successive saddle maps is not zero (although it is not difficult to see that all compositions are homotopic to zero).

In order to obtain a chain complex and so complete the proof of the main theorem, we thicken the FK sequence by contractible pieces. Specifically, we consider the truncated Frenkel-Khovanov sequence $\mathrm{FK}_{n, l}$ of length $l$ and our construction is inductive in $l$.

Let $P_{n-1} \in \operatorname{Kom}(n-1)$ be a chain complex representing the $(n-1)$ st universal projector. We will now define a chain complex $\mathrm{CFK}_{n, l}$ inductively in length $l$ using the maps $\left\{f_{k}\right\}_{0}^{\infty}$ of the FK-sequence. At each stage $\mathrm{CFK}_{n, l}$ is defined as either a two term chain complex using $f_{l-1}$ (in case the following map $f_{l}$ has $q$-degree 1 ) or as a three term chain complex using $f_{l-1}$ and $f_{l}$ (in case $f_{l}$ has degree 2 ).

Definition 7.9. Set $\mathrm{CFK}_{n, 0}=P_{n-1} \sqcup 1$. For each $l>0$ the $q$-degree of $f_{l-1}$ is either 1 or 2 . If $f_{l-1}$ has $q$-degree 1 then set $\mathrm{CFK}_{n, l}$ equal to

$$
\begin{cases}\mathrm{CFK}_{n, l-1} \stackrel{f_{l-1}}{\longrightarrow} q f_{l-1} \mathrm{CFK}_{n, l-1} & \text { if } \operatorname{deg}_{q}\left(f_{l}\right)=1, \\ \mathrm{CFK}_{n, l-1} \stackrel{f_{l-1}}{\longrightarrow} q f_{l-1} \mathrm{CFK}_{n, l-1} \stackrel{f_{l}}{\longrightarrow} q^{3} f_{l} f_{l-1} \mathrm{CFK}_{n, l-1} & \text { if } \operatorname{deg}_{q}\left(f_{l}\right)=2 .\end{cases}
$$

Otherwise, the $q$-degree of $f_{l-1}$ is 2 and we set

$$
\mathrm{CFK}_{n, l}=\mathrm{CFK}_{n, l-1} \text {. }
$$

In this second step we do not change the complex $\mathrm{CFK}_{n, l}$ after having just used a degree 2 map in order to avoid a degree shift.

Here we follow the convention that

$$
\begin{aligned}
f_{l}(D \oplus D) & =f_{l}(D) \oplus f_{l}(D), \\
q^{i}\left(q^{j} D\right) & =q^{i+j} D,
\end{aligned}
$$

and

$$
f_{l}(D)=D
$$


if $\operatorname{deg}_{q}\left(f_{l}\right)=2$. Note that the three term sequence in the last case is indeed a chain complex, that is $f_{l} f_{l-1}=0$.

The recursive step can be visualized as follows. (1) If $\operatorname{deg}_{q}\left(f_{l-1}\right)=1$ and $\operatorname{deg}_{q}\left(f_{l}\right)=1$, then we have

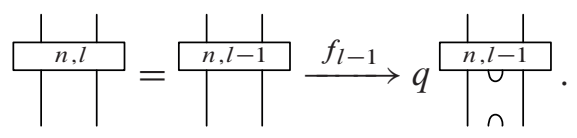

(2) If $\operatorname{deg}_{q}\left(f_{l}\right)=2$, then we have

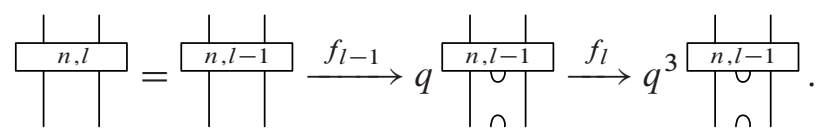

Consider the chain complex $\mathrm{CFK}_{4,3}$ :

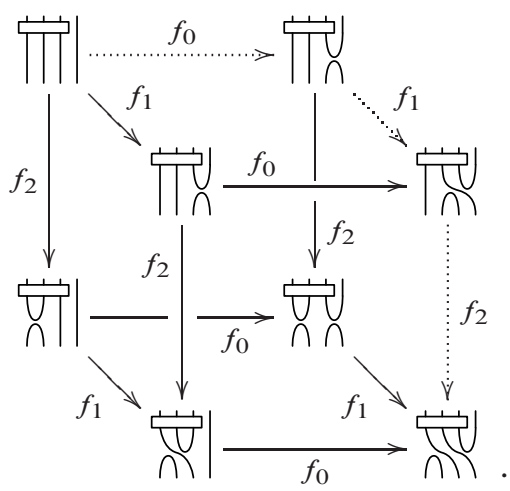

(To make the illustration more compact, only a part of the actual chain complex $\mathrm{CFK}_{4,3}$ is shown above. The actual $\mathrm{CFK}_{4,3}$ has another layer below the cube shown above.)

Recall that the truncated Frenkel-Khovanov sequence $\mathrm{FK}_{4,3}$ is given by

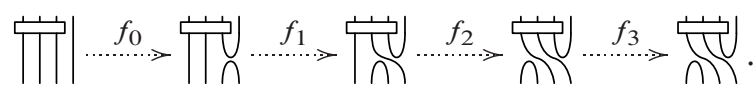

Here we use dotted arrows to help the reader find the relevant information. The first four terms of the sequence $\mathrm{FK}_{4,3}$ start in the upper left hand corner of the cube, travel to the right then to the front face of the cube and land in the lower right hand corner. These are precisely the first four terms of the diagram pictured at the beginning of Section 7.2 together with four contractible terms.

The proofs contained in the remainder of this section are rooted in the observation that $\mathrm{CFK}_{n, l}$ will always decompose as $\mathrm{FK}_{n, l}$ plus a contractible subcomplex $K_{l}$ consisting of truncated Frenkel-Khovanov sequences containing turnbacks. 
Lemma 7.10 (structure of $\mathrm{CFK}_{n, l}$ ). For each $l \geq 0$ the chain complex $\mathrm{CFK}_{n, l}$ admits a decomposition,

$$
\mathrm{CFK}_{n, l} \cong\left(P_{n-1} \sqcup 1\right) \otimes\left(\mathrm{FK}_{n, l} \oplus K_{l}\right),
$$

where the second summand $\left(P_{n-1} \sqcup 1\right) \otimes K_{l}$ is contractible.

More specifically, the contractibility of $\left(P_{n-1} \sqcup 1\right) \otimes K_{l}$ is a consequence of simultaneous Gaussian elimination of some of the terms in $K_{l}$, so that each remaining term (in the notation of Lemma 2.11) $\left(P_{n-1} \sqcup 1\right) \otimes C_{i}$ is contractible. Moreover, the off-diagonal component

$$
\left(P_{n-1} \sqcup 1\right) \otimes K_{l} \stackrel{\beta}{\longrightarrow}\left(P_{n-1} \sqcup 1\right) \otimes \mathrm{FK}_{n, l}
$$

of the $\mathrm{CFK}_{n, l}$ differential with respect to the decomposition (2) vanishes on the domain of the isomorphisms underlying the simultaneous Gaussian elimination in $K_{l}$.

Proof. The proof is by induction on $l$ using the recurrence defining $\mathrm{CFK}_{n, l}$ (Definition 7.9). If $l=0$ or $\operatorname{deg}_{q}\left(f_{l-1}\right)=2$ then there is nothing to prove. If $\operatorname{deg}_{q}\left(f_{l}\right) \neq 2$ then $\mathrm{CFK}_{n, l}$ is defined as a two term sequence,

$$
\mathrm{CFK}_{n, l}=\mathrm{CFK}_{n, l-1} \stackrel{f_{l-1}}{\longrightarrow} q f_{l-1} \mathrm{CFK}_{n, l-1} .
$$

By induction we may assume that

$$
\mathrm{CFK}_{n, l-1} \cong\left(P_{n-1} \sqcup 1\right) \otimes\left(\mathrm{FK}_{n, l-1} \oplus K_{l-1}\right),
$$

where $K_{l-1} \simeq 0$ satisfies the conclusion of the lemma. We claim that there is a decomposition

$$
\mathrm{CFK}_{n, l} \cong\left(P_{n-1} \sqcup 1\right) \otimes\left(\mathrm{FK}_{n, l} \oplus q f_{l-1} \mathrm{FK}_{n, l-2} \oplus K_{l-1} \oplus q f_{l-1} K_{l-1}\right) .
$$

This can be observed by writing the most important part of the recursion defining $\mathrm{CFK}_{n, l}$ in a helpful way:

$$
\begin{gathered}
{\left[\mathrm{FK}_{n, l-1}(0) \stackrel{f_{0}}{\longrightarrow} \cdots \stackrel{f_{l-3}}{\longrightarrow} \mathrm{FK}_{n, l-1}(l-2) \stackrel{f_{l-2}}{\longrightarrow} \mathrm{FK}_{n, l-1}(l-1)\right]} \\
{\left[f_{l-1} f_{l-1} \mathrm{FK}_{n, l-1}(0) \underset{f_{0}}{\longrightarrow} \cdots \underset{f_{l-3}}{\longrightarrow} f_{l-1} \mathrm{FK}_{n, l-1}(l-2)\right] \underset{\beta_{1}}{\longrightarrow} f_{l-1} \mathrm{FK}_{n, l-1}(l-1)} \\
\| f_{l-1} \\
\mathrm{FK}_{n, l}(l) .
\end{gathered}
$$

The $i$ th row above is contained in the $i$ th term below, $i=1,2$ :

$$
\mathrm{CFK}_{n, l-1} \stackrel{f_{l-1}}{\longrightarrow} f_{l-1} \mathrm{CFK}_{n, l-1} \text {. }
$$


In this diagram the lower order projector $\left(P_{n-1} \sqcup 1\right)$ is omitted to simplify the notation. The terms in the top row are $\mathrm{FK}_{n, l-1}$, the truncated Frenkel-Khovanov sequence of length $l-1$. By the inductive assumption, this sequence is a summand in $\mathrm{CFK}_{n, l-1}$ and the remaining part - the contractible summand $K_{l-1}-$ is not included in the diagram. The terms on the left in the bottom row are of the form $f_{l-1} \mathrm{FK}_{n, l-2}$. Observe that by definition the last term $f_{l-1} \mathrm{FK}_{n, l-1}(l-1)$ is equal to the next term $\mathrm{FK}_{n, l}(l)$ in the Frenkel-Khovanov sequence. The $\mathrm{FK}_{n, l}$ summand in (2) is seen in the diagram above as $\mathrm{FK}_{n, l-1}$ in the top row followed by the vertical map $f_{l-1}$ to $f_{l-1} \mathrm{FK}_{n, l-1}(l-1)$. Equation (3) follows immediately.

To prove that $\left(P_{n-1} \sqcup 1\right) \otimes K_{l}$ is contractible, where

$$
K_{l}=q f_{l-1} \mathrm{FK}_{n, l-2} \oplus K_{l-1} \oplus q f_{l-1} K_{l-1},
$$

further analysis of the differential $\mathrm{CFK}_{n, l}$ is necessary. Note that $\left(P_{n-1} \sqcup 1\right) \otimes$ $f_{l-1} \mathrm{FK}_{n, l-2}$ is contractible by Corollary 7.6, more precisely all terms in this sequence are either contained in triples or quadruples or are projectors capped by turnbacks. By the inductive hypothesis on the off-diagonal entry of the differential in the statement of the lemma, when the terms participating in the Gaussian eliminations in $K_{l-1}, q f_{l-1} K_{l-1}$ and $q f_{l-1} \mathrm{FK}_{n, l-2}$ are grouped together, (in the notation of Lemma 2.11) the isomorphisms underlying the Gaussian eliminations in the summands, $a_{2 i}: A_{2 i} \rightarrow A_{2 i+1}$ and $e_{2 i+1}: B_{2 i+1} \rightarrow B_{2 i+2}$ remain isomorphisms because the matrices are lower triangular. After removing all triples and quadruples, the remaining chain complex consisting of contractible terms may be contracted by Lemma 2.12 (big collapse). This concludes the proof that $\left(P_{n-1} \sqcup 1\right) \otimes K_{l}$ is contractible.

To propagate the inductive hypothesis on the differential, note that the only new off-diagonal component, of the form in the statement of the lemma, introduced during the inductive step, is the map $\beta_{l}$ in the diagram above. The Gaussian eliminations take place in the sequence $q f_{l-1} \mathrm{FK}_{n, l-2}$ in the bottom row and since $\beta_{l}$ is defined on the last term of that sequence, clearly the component of the differential on the domain of the isomorphisms in the Gaussian eliminations is trivial.

The proof in the second case (when $\operatorname{deg}_{q}\left(f_{l}\right)=2$ ) is almost exactly the same. Instead of one row of contractible terms there are two new rows of contractible terms. By definition,

$$
\mathrm{CFK}_{n, l}=\mathrm{CFK}_{n, l-1} \stackrel{f_{l-1}}{\longrightarrow} q f_{l-1} \mathrm{CFK}_{n, l-1} \stackrel{f_{l}}{\longrightarrow} q^{3} f_{l} f_{l-1} \mathrm{CFK}_{n, l-1} .
$$

Again by induction we may assume that

$$
\mathrm{CFK}_{n, l-1} \cong\left(P_{n-1} \sqcup 1\right) \otimes\left(\mathrm{FK}_{n, l-1} \oplus K_{l-1}\right)
$$

and $C_{l-1} \simeq 0$. In this case the claim is that there is a decomposition

$$
\mathrm{CFK}_{n, l} \cong\left(P_{n-1} \sqcup 1\right) \otimes\left(\mathrm{FK}_{n, l} \oplus K_{l}\right),
$$


where

$$
K_{l}=q f_{l-1} \mathrm{FK}_{n, l-2} \oplus K_{l-1} \oplus q f_{l-1} K_{l-1} \oplus q^{3} f_{l} f_{l-1} \mathrm{FK}_{n, l-1} \oplus q^{3} f_{l} f_{l-1} K_{l-1} .
$$

Again this can be observed by writing the most important part of the recursion as follows:

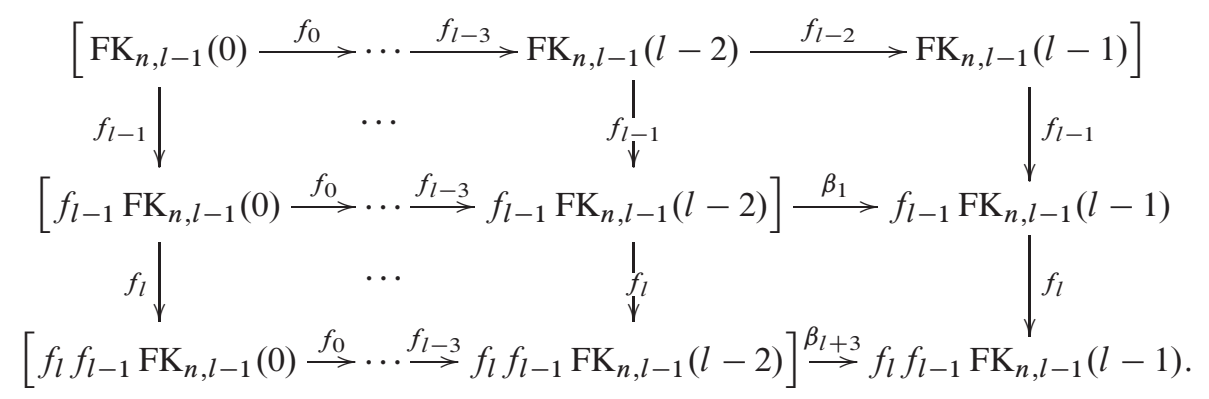

The $i$ th row above is contained in the $i$ th term below, $i=1,2,3$ :

$$
\mathrm{CFK}_{n, l-1} \stackrel{f_{l}}{\longrightarrow} f_{l} \mathrm{CFK}_{n, l-1} \stackrel{f_{l+1}}{\longrightarrow} f_{l+1} f_{l} \mathrm{CFK}_{n, l-1} \text {. }
$$

As in the previous case, the summands in $K_{l}$ are contractible.

Remark 7.11. The proof of the lemma above used the recursive definition of the chain complex $\mathrm{CFK}_{n, l}$. Completely expanding the recursion gives the following decomposition:

$$
\mathrm{CFK}_{n, l}=\bigoplus_{I} q^{l(I)+\tau(I)} f_{I} \mathrm{FK}_{n, l-l(I)}
$$

where $I$ are $k$-tuples indexing maps in the sequence $\mathrm{FK}_{n}, l(I)$ is the cardinality of $I, f_{I}=f_{i_{1}} \circ f_{i_{2}} \circ \cdots \circ f_{i_{k}}$ when $I=\left(i_{1}, i_{2}, \ldots, i_{k}\right)$ and $\tau(I)$ is the number of degree 2 maps in $f_{I}$. Moreover, if $f_{m}$ is the differential of $\mathrm{FK}_{n}$ then the differential of in each summand, $f_{I} \mathrm{FK}_{n, l-l(I)}$, is $f_{I}\left(f_{m}\right)$. Each summand (except for $I=\emptyset$ ) is contractible by Corollary 7.6. In the notation of Lemma 7.10, $K_{l}=\bigoplus_{I \neq \emptyset} q^{l(I)+\tau(I)} f_{I} \mathrm{FK}_{n, l-l(I)}$ and the summand $\mathrm{FK}_{n, l}$ corresponds to $I=\emptyset$.

The following statement is important for establishing the properties of a universal projector:

Lemma 7.12 (contractibility under turnbacks). Let $n>2, l \geq 0$ and $j \in\{1, \ldots$, $n-1\}$. Then all terms in the chain complex

$$
\mathrm{CFK}_{n, l} \otimes e_{j}
$$

may be contracted, except possibly for the lth term,

$$
\left(P_{n-1} \sqcup 1\right) \otimes \mathrm{FK}_{n, l}(l)
$$


Proof. By Lemma 7.10,

$\mathrm{CFK}_{n, l} \otimes e_{j} \cong\left(\left(P_{n-1} \sqcup 1\right) \otimes\left(\mathrm{FK}_{n, l} \otimes e_{j}\right)\right) \oplus\left(\left(P_{n-1} \sqcup 1\right) \otimes\left(K_{l} \otimes e_{j}\right)\right)$.

Application of $-\otimes e_{j}$ does not change the contractibility of the second summand. By Proposition 7.5 all of the terms (besides possibly the last, depending on $j$ ) in the first summand are either projectors $P_{n-1}$ capped by turnbacks or contained in triples and quadruples. The rest of the proof is identical to the proof of the inductive step in Lemma 7.10.

As a consequence of Lemma 7.10 we have the following definition.

Definition 7.13 (the truncated projector $P_{n, l}$ ). Contracting $K_{l} \subset \mathrm{CFK}_{n, l}$ yields a homotopy equivalence

$$
\mathrm{CFK}_{n, l} \longrightarrow P_{n, l}
$$

onto a chain complex $P_{n, l}$ that consists of the first $l$ terms of the Frenkel-Khovanov sequence pictured at the beginning of Section 7.2.

Note that the chain complex $P_{n, l}$ may be thought of as a completed version of the truncated homotopy chain complex $\mathrm{HP}_{n, l}$ (Definition 7.7). Given a homotopy chain complex there is a standard obstruction theoretic approach to constructing a chain complex in which new components corresponding to nullhomotopies and Massey products of nullhomotopies are added to the differential; see IV.2.10 in [10]. The extra maps in $P_{n, l}$ are precisely those corresponding to these homotopies and Massey products. Our axioms (Definition 3.1) guarantee that any such choice of Massey products yields a unique chain complex up to homotopy.

The universal projector $P_{n}$ will be defined as the limit of $P_{n, l}$ as $l \rightarrow \infty$. Its contractibility under turnbacks (to show that $P_{n}$ satisfies the axioms of a universal projector in Definition 3.1) follows from Lemma 7.12. The remaining property, ensuring that the limit exists, is the "stability" of the sequence $\left\{P_{n, l}\right\}$, proved in the following Proposition 7.14.

We now show that the chain complex $P_{n, l+1}$ is obtained from the chain complex $P_{n, l}$ by adding the next term in the picture at the beginning of Section 7.2 and only adding maps to the differential from the old terms to the new term. The maps between those terms in $P_{n, l+1}$ which come from $P_{n, l}$ are exactly the same as the maps between terms in $P_{n, l}$. We may conclude from this together with the previous proposition that there is a chain complex $P_{n}=P_{n, \infty}$ which is a universal projector.

Proposition 7.14 (Stability of construction). The inclusion

$$
P_{n, l} \hookrightarrow P_{n, l+1}
$$

is an isomorphism onto its image. Moreover,

$$
P_{n, l+1} \cong P_{n, l} \oplus\left(\left(P_{n-1} \sqcup 1\right) \otimes \mathrm{FK}_{n, l}(l)\right) .
$$


$d_{P_{n, l+1}}$ is lower triangular with respect to this decomposition and $\left.d_{P_{n, l+1}}\right|_{P_{n, l}}=$ $d_{P_{n, l}}$.

Proof. This follows from an analogue of the first commutative diagram in the proof of Proposition 7.10. $P_{n, l+1}$ is obtained from $P_{n, l}$ by

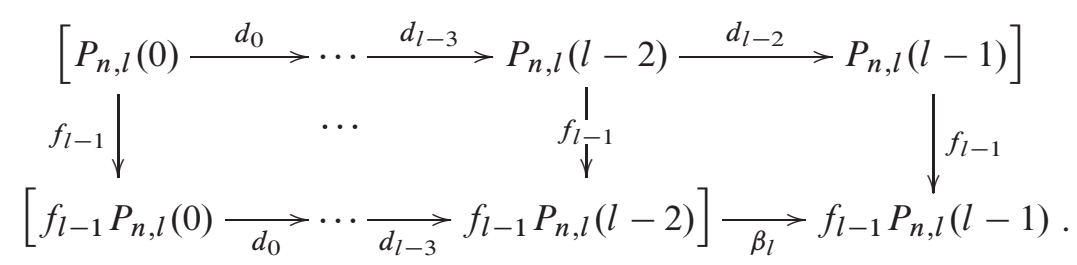

For the sake of clarity we have omitted from the diagram the parts of the differential $d_{P_{n, l}}$ between non-consecutive terms. The terms in the lower left-hand corner are again contractible. Contracting them does not change the maps $d_{i}$ along the top row.

7.4. A doubling construction. In the proof of the main theorem we only concerned ourselves with what could be called right contractibility or the statement that for $C_{*} \in \operatorname{Kom}(n)$ and $0<i<n$,

$$
C_{*} \otimes e_{i} \simeq 0 .
$$

If $C_{*}$ is right contractible then define $\bar{C}_{*} \in \operatorname{Kom}(n)$ to be the chain complex in which each diagram and morphism is flipped upside down. Now define a new chain complex $D_{*}$ by

$$
D_{*}=\bar{C}_{*} \otimes C_{*} .
$$

The contractibility of $D_{*}$ by turnbacks on both sides now follows from that of $C_{*}$ on one side. The first two axioms of the universal projector are satisfied by $D_{*}$ provided that they are satisfied by $C_{*}$.

\section{References}

[1] J. Bernstein, I. Frenkel, and M. Khovanov, A categorification of the Temperley-Lieb algebra and Schur quotients of $\mathrm{U}(\mathfrak{s l}(2))$ via projective and Zuckerman functors. Sel. Math., New Ser. 5 (1999), 199-241. MR 1714141 Zbl 0981.17001

[2] C. Blanchet, N. Habegger, G. Masbaum, and P. Vogel, Topological quantum field theories derived from the Kauffman bracket. Topology 34 (1995), 883-927. MR 1362791 Zbl 0887.57009

[3] B. Bakalov and A. Kirillov Jr, Lectures on tensor categories and modular functors. Amer. Math. Soc., Providence (RI), 2001. MR 1797619 Zbl 0965.18002

[4] D. Bar-Natan, Khovanov's homology for tangles and cobordisms. Geom. Topol. 9 (2005), 1443-1499. MR 2174270 Zbl 1084.57011 www.msp.warwick.ac.uk/gt/2005/09/p033.xhtml 
[5] A. Beliakova and S. Wehrli, Categorification of the colored Jones polynomial and Rasmussen invariant of links. Can. J. Math. 60 (2008), 1240-1266. MR 2462446 Zbl 1171.57010

[6] B. Elias, A diagrammatic Temperley-Lieb categorification. Int. J. Math. Math. Sci. 2010 (2010), Art. ID 530808. MR 2726291 Zbl 05825739 www.hindawi.com/journals/ijmms/2010/530808/

[7] I. Frenkel and M. Khovanov, Canonical bases in tensor products and graphical calculus for $\mathrm{U}_{q} \mathfrak{s l}(2)$. Duke Math. J. 87 (1997), 409-480. MR 1446615 Zbl 0883.17013

[8] I. Frenkel, M. Khovanov, and C. Stroppel, A categorification of finite-dimensional irreducible representations of quantum $\mathrm{U}_{q} \mathfrak{s l}(2)$ and their tensor products. Sel. Math., New Ser. 12 (2006), 379-431. MR 17B37 Zbl 1188.17011

[9] I. Frenkel, C. Stroppel, and J. Sussan, Categorifying fractional Euler characteristics, Jones-Wenzl projector and 3j-symbols. Quantum Topol. 3 (2012), 181-253.

[10] S. I. Gelfand and Y. I. Manin, Methods of homological algebra. Springer Verlag, Berlin, 2003, second ed. MR 1950475 Zbl 1006.18001

[11] J. E. Grigsby and S. M. Wehrli, On the colored Jones polynomial, sutured Floer homology and knot Floer homology. Adv. Math. 223 (2010), 2114-2165. MR 2601010 Zbl 1205.57015

[12] M. Jacobsson, An invariant of link cobordisms from Khovanov homology. Algebr. Geom. Topol. 4 (2004), 1211-1251. MR 2113903 Zbl 1072.57018 www.msp.warwick.ac.uk/agt/2004/04/p053.xhtml

[13] V. F. R. Jones, A polynomial invariant for knots via von Neumann algebras. Bull. Am. Math. Soc., New Ser. 12 (1985), 103-111. MR 766964 Zbl 0564.57006 www.ams.org/journals/bull/1985-12-01/S0273-0979-1985-15304-2/home.html

[14] L. H. Kauffman and S. L. Lins, Temperley-Lieb recoupling theory and invariants of 3-manifolds. Princeton University Press, Princeton (NJ), 1994. MR 1280463 Zbl 0821.57003

[15] M. Khovanov, A categorification of the Jones polynomial. Duke Math. J. 101 (2000), 359-426. MR 1740682 Zbl 0960.57005

[16] M. Khovanov, A functor-valued invariant of tangles. Algebr. Geom. Topol. 2 (2002), 665-741. MR 1928174 Zbl 1002.57006 www.msp.warwick.ac.uk/agt/2002/02/p030.xhtml

[17] M. Khovanov, Categorifications of the colored Jones polynomial. J. Knot Theory Ramifications 14 (2005), 111-130. MR 2124557 Zbl 1083.57019

[18] M. Khovanov, Link homology and Frobenius extensions. Fundam. Math. 190 (2006), 179-190. MR 2232858 Zbl 1101.57004

[19] M. Khovanov, V. Mazorchuk, and C. Stroppel, A brief review of abelian categorifications. Theory Appl. Categ. 22 (2009), 479-508. MR 2559652 Zbl 1181.18005

[20] G. Kuperberg, Spiders for rank 2 Lie algebras. Commun. Math. Phys. 180 (1996), 109-151. MR 1403861 Zbl 0870.17005

[21] W. B. R. Lickorish, An introduction to knot theory. Springer-Verlag, New York (NY), 1997. MR 1472978 Zbl 0886.57001 
[22] S. Morrison and A. Nieh, On Khovanov's cobordism theory for $\mathrm{U}_{q} \mathfrak{s u}(3)$ knot homology. J. Knot Theory Ramifications 17 (2008), 1121-1173. MR 2457839 Zbl 05375320

[23] L. Rozansky, An infinite torus braid yields a categorified jones-wenzl projector. Preprint 2010. arXiv:1005.3266

[24] C. Stroppel, Categorification of the Temperley-Lieb category, tangles, and cobordisms via projective functors. Duke Math. J. 126 (2005), 547-596. MR 2120117 Zbl 1112.17010

[25] V. G. Turaev, Quantum invariants of knots and 3-manifolds. Walter de Gruyter, Berlin, 1994. MR 1292673 Zbl 0812.57003

[26] V. G. Turaev and O. Ya. Viro, State sum invariants of 3-manifolds and quantum $6 j$-symbols. Topology 31 (1992), 865-902. MR 1191386 Zbl 0779.57009

[27] K. Walker, On Witten's 3-manifold invariants. Preprint 1991. canyon23.net/math/

[28] C. Weibel, The K-book: An introduction to algebraic K-theory. Book in preparation. Preprint 2011. www.math.rutgers.edu/ weibel/Kbook.html

[29] H. Wenzl, On sequences of projections. C. R. Math. Acad. Sci., Soc. R. Can. 9 (1987), 5-9. MR 873400 Zbl 0622.47019

Received July 14, 2010

Benjamin Cooper, Department of Mathematics, University of Virginia, Charlottesville, VA 22904, U.S.A.

E-mail: bjc4n@virginia.edu

Vyacheslav Krushkal, Department of Mathematics, University of Virginia, Charlottesville, VA 22904, U.S.A.

E-mail: krushkal@virginia.edu 